\title{
HIGH ANGULAR RESOLUTION IMAGING SPECTROSCOPY OF THE GALACTIC ULTRACOMPACT H II REGION K3-50A
}

\author{
Robert D. Blum ${ }^{1}$ And Peter J. McGregor ${ }^{2}$ \\ ${ }^{1}$ NOAO Gemini Science Center, 950 North Cherry Avenue, Tucson, AZ 85719, USA \\ ${ }^{2}$ Research School of Astronomy and Astrophysics, The Australian National University, Cotter Road, Weston Creek, ACT 2611, Australia \\ Received 2008 October 31; accepted 2009 June 8; published 2009 June 29
}

\begin{abstract}
Gemini North adaptive optics (AO) imaging spectroscopy is presented for the Galactic ultracompact $\mathrm{H}$ II (UCH II) region K3-50A. Data were obtained in the $K$ band using the Near-infrared Integral Field Spectrograph (NIFS) behind the facility AO module ALTAIR in natural guide star mode. The NIFS data cube reveals a complex spatial morphology across the $0.1 \mathrm{pc}$ scale of the $3^{\prime \prime} \mathrm{UCH}$ II region. Comparison of the nebular emission to Cloudy ionization models shows that the central source must have an effective temperature between about $37,000 \mathrm{~K}$ and $45,000 \mathrm{~K}$ with preferred values near $40,000 \mathrm{~K}$. Evidence is presented for sharp density variations in the nebula which are interpreted as a clearing of material nearest the central source. High excitation lines of Fe III and Se IV show that the ionization of the nebula clearly changes with distance from the central source. A double lobed kinematic signature $\left( \pm 25 \mathrm{~km} \mathrm{~s}^{-1}\right)$ is evident in the $\mathrm{Br} \gamma$ line map which may be related to the larger scale ionized flow detailed in earlier investigations. This signature and the large-scale flow are not co-aligned, but they may still be related. Though clearly resolved from the nebula, the central source itself remains buried, and the NIFS spectrum shows no evidence of photospheric lines.
\end{abstract}

Key words: dust, extinction - H II regions - infrared: stars - instrumentation: adaptive optics - stars: formation

Online-only material: color figures

\section{INTRODUCTION}

K3-50A has been the subject of many studies in the optical/ near-infrared and radio regimes and most wavelengths in between. It is highly obscured at short wavelengths and bright at long wavelengths. The object was originally part of the planetary nebulae survey of Kohoutek (1965), but was later reclassified as an H II region. Persson \& Frogel (1974) observed K3-50A at mid-infrared wavelengths and showed the optical nebula was offset from the radio source due to a strong gradient in the foreground extinction. Wynn-Williams et al. (1977) determined precise offsets between the radio, infrared, and optical sources which validate the Persson \& Frogel (1974) model.

K3-50A, also known as the Galactic radio source G70.3-1.6, has been extensively observed at long wavelengths. Turner \& Matthews (1984) provided a high angular resolution map at $2 \mathrm{~cm}$ which resolved the ionized gas into a clumpy arclike or shelllike structure on scales smaller than $1^{\prime \prime}$. Kurtz et al. (1994) included this object in their catalog of UCH II regions, describing it as a core-halo type while De Pree et al. (1994) observed the larger nebula in the continuum and radio recombination line $\mathrm{H} 76 \alpha$. The radio morphology is extended in two lobes to the NW and SE and De Pree et al. (1994) found a velocity gradient along the lobes which led them to suggest the structure was an ionized outflow extending tens of arcseconds from the center of the UCH II region.

Howard et al. (1996) presented $\mathrm{Br} \gamma$ and $\mathrm{Br} \alpha$ emission-line maps at greater than arcsecond resolution and followed this work (Howard et al. 1997) with molecular line maps which they modeled as arising from a large-scale molecular torus (roughly perpendicular to the ionized outflow of De Pree et al. 1994).

More recent work has centered on near-infrared and midinfrared wavelengths and higher angular resolution data. Hofmann et al. (2004) observed the nebula in the $K$-band using speckle techniques to provide an order of magnitude higher angular resolution than previous near-infrared work, and Okamoto et al. (2003) used the Subaru $8 \mathrm{~m}$ telescope and mid-infrared imager COMICS to obtain $\lesssim 0$ ' 5 resolution images and spectra near $10 \mu \mathrm{m}$.

In this paper, we present $K$ band integral-field spectroscopy of K3-50A obtained on the Gemini North $8 \mathrm{~m}$ telescope with the Near-infrared Integral Field Spectrograph (NIFS). These data were taken in conjunction with the facility adaptive optics (AO) system and result in the most detailed data cube yet for the core of K3-50A. In an earlier paper (Blum \& McGregor 2008), we presented similar data for the UCH II region G45.45+0.6. In that case, several massive stars were identified by photospheric lines and other objects showed signatures of buried massive young stellar objects. G45.45+0.6 clearly harbors a rich cluster. Messineo et al. (2007) make similar conclusions based on their ESO VLT SINFONI integral-field spectrograph observations for the UCH II region in the cluster [DBS2003]8. Previous investigators have suggested the same may be true of K3-50A: Colgan et al. (1991), Okamoto et al. (2003), and Hofmann et al. (2004). Our data identify multiple continuum sources in K3$50 \mathrm{~A}$ and support the multiple star model, but we also show that the excitation in the central few arcseconds of $\mathrm{K} 3-50 \mathrm{~A}$ is likely dominated by a single object (the unresolved continuum peak).

In the following, the distance to the nebula is based on the work of Harris (1975). The Harris (1975) distance is derived from a compilation of velocities and a rotation model; Harris' distance needs to be reduced due to a revised distance to the Galactic center (Harris 1975, used a distance of $10 \mathrm{kpc}$ ). Adopting a value of $8 \mathrm{kpc}$ gives a reduction by a factor of 0.8 resulting in a distance to the $\mathrm{H}$ II region of about $7 \mathrm{kpc}$.

\section{OBSERVATIONS}

Data were obtained with the NIFS at the Cassegrain focus of the Gemini North Fredrick C. Gillett $8 \mathrm{~m}$ telescope on Mauna Kea, Hawaii on the night of 200620 July (Hawaiian Standard 
Time). NIFS was used with the facility AO module $\mathrm{ALTAIR}^{3}$ in natural guide star (NGS) mode.

NIFS is fully described by McGregor et al. (2003); see also Blum \& McGregor (2008) for a more detailed description of similar observations with NIFS as described here. Briefly, NIFS slices an approximately three arc second by three arc second field into 29 spectral segments of 0 ". 1 width (the "slit" width) and $\sim 3^{\prime \prime}$ in length. The scale along the spatial dimension is 0 .'043 $\mathrm{pix}^{-1}$. The resulting "spaxels," or spatial pixels, are thus $0 . .043 \times 0$.' 1 in size, and each contains a full spectrum covering one of the near-infrared bands.

In the present paper, $K$-band spectra are presented for the Galactic UCH II region K3-50A. The AO guide star used for ALTAIR is located 10.'2 to the south and west of K3-50A and has an $R$ magnitude of 12.7 according to the USNO catalog (source id: U1200_14213688). There was thin cirrus at the time of the observations, and the seeing (at $5000 \AA$, corrected to zenith) reported during the observations by ALTAIR was approximately 0.45 . The observations were obtained at relatively high air mass (1.5), and ALTAIR was run at $500 \mathrm{~Hz}$.

The observations consisted of a single co-added frame taken on source and a second frame obtained on a nearby ( $\sim 50^{\prime \prime}$ west) blank field. Each frame had a total exposure time of $600 \mathrm{~s}(15$ $\times 40$ s) on source and 600 s on sky. NIFS was oriented with a position (P.A.) of $70^{\circ}$ east of north to correspond approximately to the orientation of the large-scale ionized flow from K3-50A (De Pree et al. 1994). This PA is aligned with the outflow direction.

The spectral resolving power of NIFS in the $K$ band is $\lambda / \Delta \lambda=$ 5160 which results in a linear dispersion of $2.13 \AA$ pixel $^{-1}$. This dispersion, combined with the large format array gives a full wavelength coverage at $K$ of about $4200 \AA$ accounting for some minor truncation in the final data cube due to the systematic shift of wavelength in each slitlet from the staircase design of the image slicer.

\section{DATA REDUCTION}

The line maps and spectra presented here were obtained using the Gemini NIFS IRAF ${ }^{4}$ data reduction package (version 1.9).

The NIFS IRAF package allows for full reduction to the "image cube" stage where a final cube has a roughly $60 \times 62$ pixel image plane and a 2040 pixel spectral depth. First, raw images are prepared for reduction by standard Gemini procedures that create the FITS image variance and data quality extensions. Next, the data are sky subtracted, flatfielded, rectified spatially, and wavelength calibrated (see Blum \& McGregor 2008, for a full description of these steps). The last two steps result in a uniform spatial-spectral trace for each image slice row and a linear wavelength scale. It is important to remember that the final IRAF data cube resamples each slitlet to two 0 '.05 pixels for convenience; the angular resolution in this dimension is still 0 !' 1 .

A lamp image of Ar and Xe lines was used to determine the dispersion along each slice and as a function of the spatial dimension of each slice. A quadratic polynomial was used in this case which produced typical uncertainties in the position of a spectral line of approximately $\pm 0.2 \AA(\sim 1 / 10$ pixel). Final spectra are interpolated to a linear wavelength solution.

\footnotetext{
3 http://www.gemini.edu/sciops/instruments/altair/altairIndex.html.

4 IRAF is distributed by the National Optical Astronomy Observatories, which are operated by the Association of Universities for Research in Astronomy, Inc., under cooperative agreement with the National Science Foundation.
}
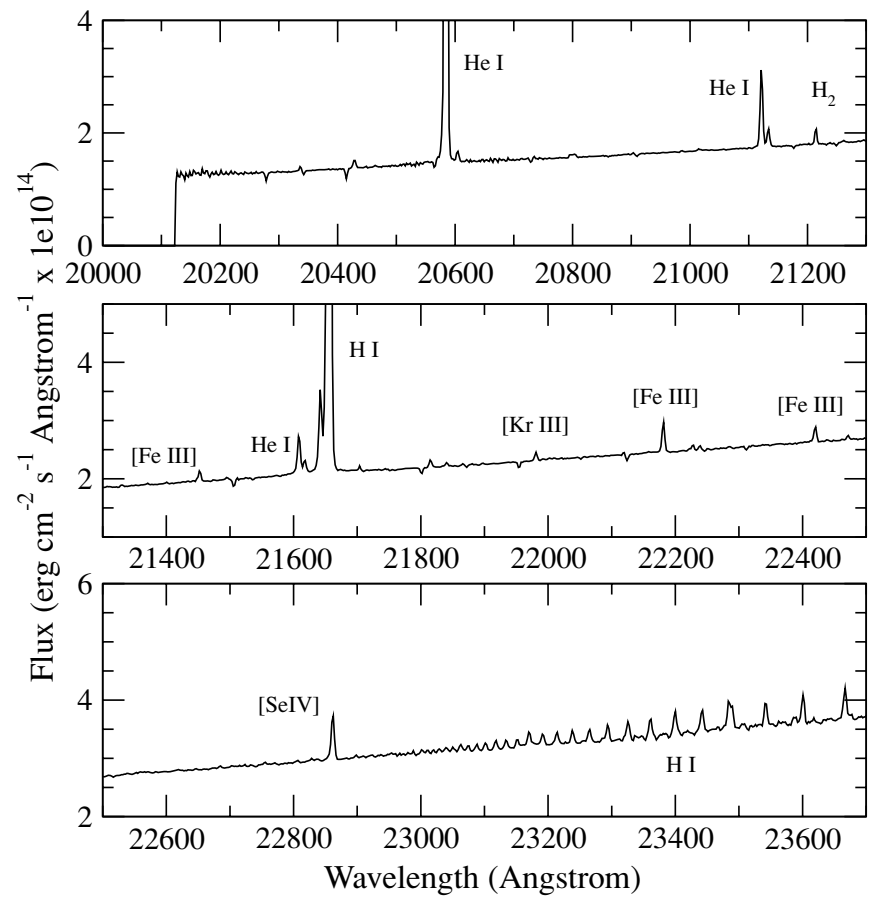

Figure 1. $K$-band spectrum extracted from the central $2 . .5$ diameter of the NIFS FOV. Differences between the object and sky frames resulted in a slight over-subtraction. Line identifications and strengths relative to $\mathrm{Br} \gamma$ are given in Table 1, and a number of the brighter lines are indicated in the figure. The $\mathrm{HI}_{\mathrm{I}}$ Pfund series is seen near $23000 \AA$ and redward.

The spectra were next corrected for telluric absorption by division by the spectrum of an A0 V star (HIP 102568). The spectrum of HIP 102568 was corrected for intrinsic $\mathrm{Br} \gamma$ absorption by fitting a Voigt profile to the telluric spectrum. Particular care was taken in the fit in order to remove this feature from the telluric standard as accurately as possible.

The final wavelength calibrated, spatially rectified, and telluric corrected images were transformed into data cubes. The spatial pixel scale was resampled to $00^{\prime} .05$ in the fine dimension and block replicated to $0{ }^{\prime} .05$ in the course dimension providing for a uniform scale as described in the previous section. Line maps presented below were extracted from these cubes using NFMAP.

The zero point of the wavelength calibration is confirmed by the positions of $\mathrm{OH}$ airglow lines in the sky image. Analysis of these lines shows the zero point is accurate to $\pm 2.5 \mathrm{~km} \mathrm{~s}^{-1}$. The calibration results in an observed blueshift of the nebular lines of approximately $3.8 \AA$ ( 1.8 pixels or $\left.-53 \mathrm{~km} \mathrm{~s}^{-1}\right)$. Lines are identified on a spectrum extracted from a 2 ". 5 diameter aperture centered on the NIFS FOV; see Figure 1. The spectrum is flux calibrated using the results of Howard et al. (1996, see below). The line positions and relative strengths compared to $\mathrm{Br} \gamma$ for this integrated spectrum are given in Table 1.

\section{RESULTS}

An image of the continuum near $21700 \AA$ is shown in Figure 2 along with the radio continuum image from De Pree et al. (1994). The continuum passband extracted was 9 pixels (19.2 $\AA$ ) wide. The appearance of sources in our continuum image is similar to the speckle $K$-band image presented by Hofmann et al. (2004) who report an angular resolution of 0.11 in their image derived from observations obtained under $1^{\prime \prime}$. 1 seeing. Hofmann et al. (2004) identify 10 point sources in their image, though some of 

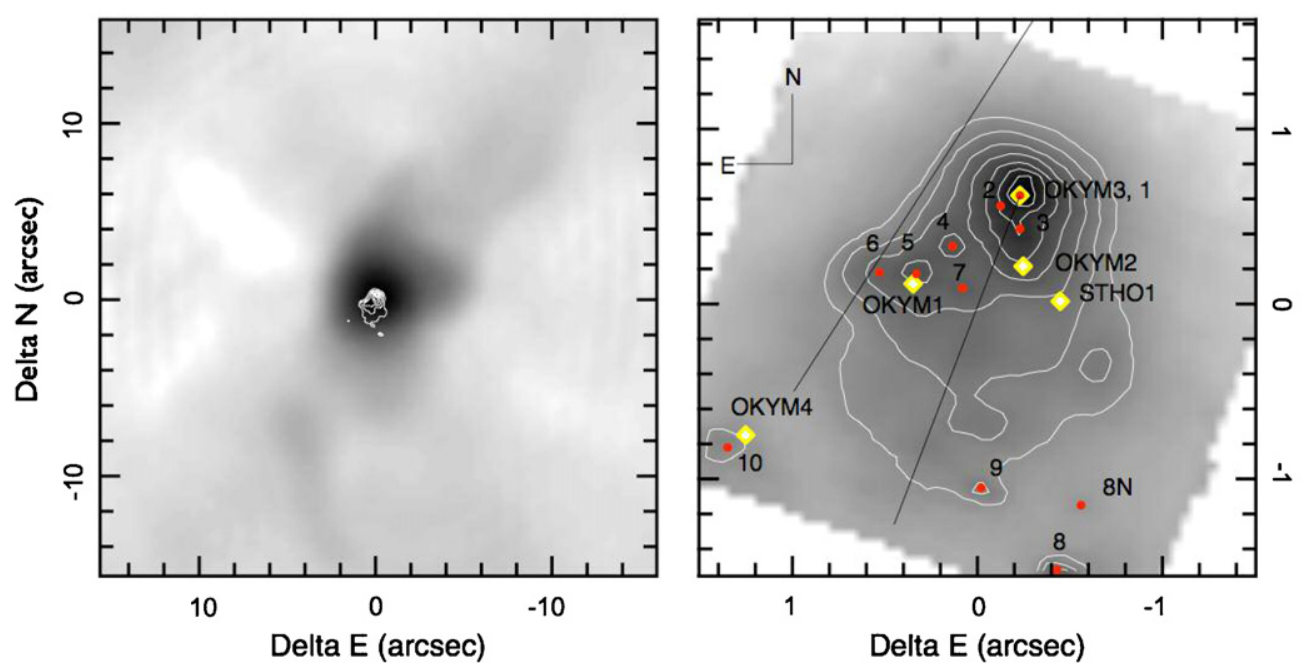

Figure 2. Continuum maps. Left panel: $\log$ of the intensity gray scale for the $14.7 \mathrm{GHz}$ radio continuum image from De Pree et al. (1994, see their Figure 1). The angular resolution is $2.0^{\prime \prime} \times 1^{\prime \prime} .6$ (beam P.A. $=79^{\circ}$ ). The contours correspond to the NIFS continuum (right panel) where the peak emission in both images is made to match. Right panel: $K$-band continuum at $21700 \AA$ (19.2 $\AA, 9$ pixels wide) shown in gray scale (log intensity) and with the same contours plotted in the left panel. The field center is located at approximately R.A. $(2000)=20^{\mathrm{h}} 01^{\mathrm{m}} 45^{\mathrm{s}} .71$, decl. $(2000)=+33^{\circ} 32^{\prime} 42^{\prime \prime}$. 1 (see the text for a discussion of source position versus wavelength). The position angle on the sky was $70^{\circ}$ (east of north); this image has been rotated so that north is up, and east is left. The red filled circles are sources from Hofmann et al. (2004) and the yellow diamonds are the mid-infrared sources of Okamoto et al. (2003); see the text. The continuum peak appears as an unresolved point source near 0.2, 0.6 and is assumed to be source 1 of Hofmann et al. (2004) and OKYM3 of Okamoto et al. (2003). The long line starting at Source 1 indicates the approximate direction of the ionizied outflow described by De Pree et al. (1994). The second line is our estimate of the rotation axis of the $\mathrm{HCO}^{+}$torus described by Howard et al. (1997); see the text. The torus is centered a few arcseconds N-W of the continuum peak.

(A color version of this figure is available in the online journal.)

Table 1

Integrated Emission-line Ratios in K3-50A

\begin{tabular}{|c|c|c|c|c|}
\hline Line Identification $^{\mathrm{a}}$ & $\lambda_{\circ}(\AA)^{\mathrm{b}}$ & $\lambda(\AA)^{\mathrm{c}}$ & FWHM $\left(\mathrm{km} \mathrm{s}^{-1}\right)$ & Ratio to $\mathrm{Br} \gamma^{\mathrm{d}}$ \\
\hline $\mathrm{H}_{2} v=1-0 \mathrm{~S}(1)$ & 21218 & 21215 & 59 & $0.0076 \pm 0.0001$ \\
\hline$[\mathrm{Fe} \mathrm{III}]{ }^{3} \mathrm{H}-{ }^{3} \mathrm{G}$ & 21457 & 21454 & 71 & $0.0052 \pm 0.0001$ \\
\hline $\mathrm{Br} \gamma\left(\mathrm{H}_{\mathrm{I}} 7-4\right)$ & 21661 & 21657 & 71 & $1.0000 \pm 0.0002$ \\
\hline$[\mathrm{Kr}$ III $]{ }^{3} \mathrm{P}_{1}-{ }^{3} \mathrm{P}_{2}$ & 21987 & 21983 & 57 & $0.0041 \pm 0.0001$ \\
\hline$[\mathrm{Fe} \mathrm{III}]{ }^{3} \mathrm{H}-{ }^{3} \mathrm{G}$ & 22184 & 22183 & 62 & $0.0164 \pm 0.0001$ \\
\hline $\mathrm{H}_{2} v=1-0 \mathrm{~S}(0)$ & 22233 & 22230 & 70 & $0.0034 \pm 0.0001$ \\
\hline$[\mathrm{Fe} \mathrm{III}]{ }^{3} \mathrm{H}-{ }^{3} \mathrm{G}$ & 22427 & 22422 & 54 & $0.0086 \pm 0.0001$ \\
\hline $\mathrm{H}_{2} v=2-1 \mathrm{~S}(1)$ & 22477 & 22473 & 55 & $0.0020 \pm 0.0001$ \\
\hline$[\mathrm{Se}$ IV $]{ }^{2} \mathrm{P}_{3 / 2}-{ }^{2} \mathrm{P}_{1 / 2}$ & 22867 & 22863 & 71 & $0.0291 \pm 0.0003$ \\
\hline
\end{tabular}

Notes.

a Atomic emission line identifications were made using the on-line data base at NIST (Ralchenko et al. 2007) and Peter van Hoof's web page (http://www.pa.uky.edu/ peter/ atomic/) that uses energy levels from the NIST database. $\mathrm{H}_{2}$ lines are from Black \& van Dishoeck (1987).

${ }^{\mathrm{b}}$ Rest wavelength, vacuum.

c Observed wavelength, mean blue shift is $-3.8 \AA$, or approximately 1.8 pixels.

${ }^{\mathrm{d}}$ Emission lines were extracted from a 2".5 diameter aperture near the center of field of view (see Figure 1). The $\mathrm{Br} \gamma$ line map is shown in Figure 3, but the line flux for this ratio was obtained by fitting multiple Gaussians to the $\mathrm{He}$ I blends and $\mathrm{Br} \gamma$; see the text.

these appear more extended than others, and this is consistent with the continuum image shown in Figure 2. The brightest source in Figure 2 is source 1 of Hofmann et al. (2004). The size of the most compact source in our image, source 8 of Hofmann et al. (2004), is about 3.7 pixels in the fine sampling direction suggesting a FWHM of $\lesssim 0{ }^{\prime} 19$. Source 1 is more extended than this in the present image ( $\sim 4.9$ pixels), but this is due to crowding with nearby blended sources and the intense nebular emission surrounding it. Hofmann et al. (2004) report source 1 to be "point like."

The 10 sources identified by Hofmann et al. (2004) are clearly seen in Figure 2 with the possible exception of their source 2 which is close to source 1 and not obvious in our image. The
Hofmann et al. (2004) sources are indicated by filled circles in Figure 2. An additional point source (named " $8 \mathrm{~N}$ ") appears near $-0.6,-1.2$ which is indicated by a single contour in Figure 2 of Hofmann et al. (2004). In addition to these objects, there are other knots or clumps which might contain embedded sources, or may be density enhancements in the $\mathrm{H}$ II region. These clumps appear to match well the morphology in Figure 1 of Hofmann et al. (2004). An exception is the "X-like" structure centered on source 1 described by Hofmann et al. (2004). We see no evidence of this feature in the lower contours of the nebular emission even though similarly bright clumps from the Hofmann et al. (2004) image do show up in our image. The mid-infrared images of Okamoto et al. (2003) also do not show this feature, though 


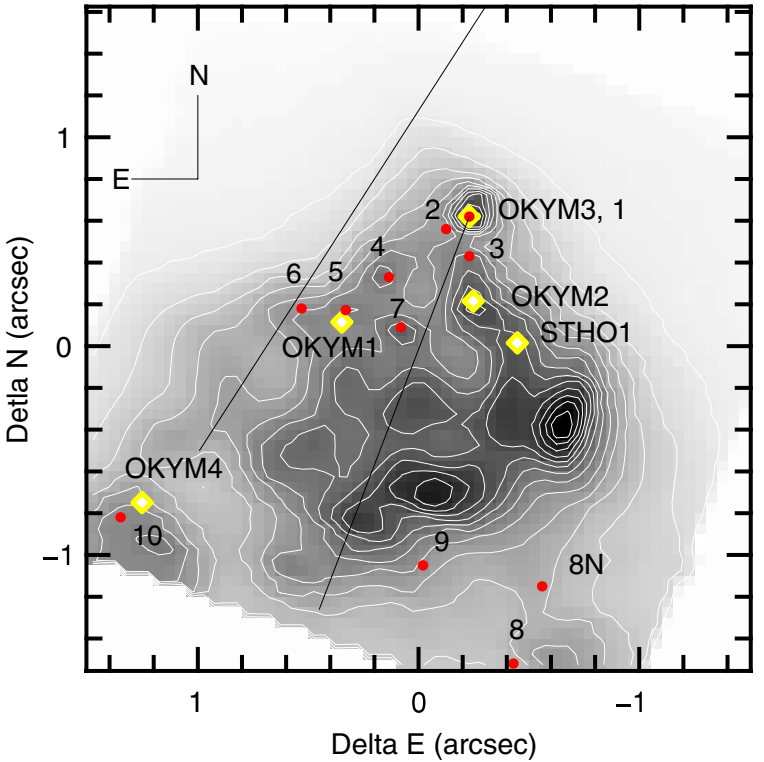

Figure 3. Continuum subtracted $\mathrm{Br} \gamma$ map. The lines and points are the same as for Figure 2. Several of the continuum sources detected in Figure 2 are among the brightest $\operatorname{Br} \gamma$ sources. The brightest peaks appear angularly resolved, and it is not clear if there are associated point sources embedded within or if these are ionized clumps of higher density material. There are 14 contours overlaid on the image. The lowest contour is $1.48 \times 10^{-13} \mathrm{erg} \mathrm{cm}^{-2} \mathrm{~s}^{-1} \operatorname{arcsec}^{-2}$ and the contour spacing is the same. The peak is located at $-0.6,-0.4$ and has a value of approximately $2.20 \times 10^{-12} \mathrm{erg} \mathrm{cm}^{-2} \mathrm{~s}^{-1} \operatorname{arcsec}^{-2}$; see the text. The $\mathrm{Br} \gamma$ line is affected by He I blends; see the text.

(A color version of this figure is available in the online journal.)

diffraction spikes from the telescope secondary are present in their images.

Okamoto et al. (2003) identified four bright sources in the core region of K3-50A covered by Figure 2 in 9-13 $\mu \mathrm{m}$ images with somewhat lower angular resolution. The three brightest, OKYM1-3, were unresolved as individual point sources. OKYM3 was associated with source 1 by Hofmann et al. (2004). The source labeled STHO1 in Figure 2 is the peak of [Ne II] emission in the maps of Okamoto et al. (2003). These authors suggest STHO1 is the location of an embedded massive star. There is no obvious point source in our continuum image or the $K$-band image of Hofmann et al. (2004) at this position.

The diamonds in Figure 2 indicate the mid-infrared sources identified by Okamoto et al. (2003). The offsets between these sources (OKYM 1-4 and STHO1) are taken from Okamoto et al. (2003) and the position of OKYM 3 is assumed to coincide with source 1 (also assumed to be the case by Hofmann et al. 2004). Hofmann et al. (2004) identified a $K$-band source with each of these Okamoto et al. (2003) sources within the positional uncertainties of the mid-infrared image. These sources are the obvious ones in Figure 2 near each of the OKYM sources.

\subsection{Line Emission Maps}

The continuum subtracted $\mathrm{Br} \gamma$ emission is shown in Figure 3. Howard et al. (1996) present narrowband Brackett imaging. Their data are seeing limited and do not resolve any of the structure shown in Figure 3. Howard et al. (1996) report a $\mathrm{Br} \gamma$ flux of $3.7 \times 10^{-12} \mathrm{erg} \mathrm{cm}^{-2} \mathrm{~s}^{-1}$ in a $3^{\prime \prime} .4$ aperture centered on the $\mathrm{Br} \gamma$ peak in their images. This covers approximately the NIFS field of view. Equating this flux to the total counts in an approximately 2 ". 5 aperture results in a peak intensity (one pixel) of $5.5 \times 10^{-15} \mathrm{erg} \mathrm{cm}^{-2} \mathrm{~s}^{-1}$ pix ${ }^{-1}$ or $2.2 \times 10^{-12} \mathrm{erg} \mathrm{cm}^{-2} \mathrm{~s}^{-1}$ $\operatorname{arcsec}^{-2}$. The peak Br $\gamma$ emission is to the SW of the continuum peak, although there is a second peak nearly as strong and very compact (point like, superposed on extended emission) centered on the continuum peak (source 1). The Hofmann et al. (2004) sources 4-7 are also centered on local $\mathrm{Br} \gamma$ peaks. The rest of the Hofmann et al. (2004) continuum sources are offset from nearby local $\mathrm{Br} \gamma$ emission peaks.

He I emission follows the $\mathrm{Br} \gamma$ emission closely. The left panel of Figure 4 shows the ratio of $\mathrm{He}$ I to $\mathrm{Br} \gamma$ for the $\mathrm{He}_{\mathrm{I}}{ }^{3} \mathrm{P}^{\circ}-{ }^{3} \mathrm{~S}$ line at $21127 \AA$. The ratio is remarkably uniform over most of the FOV and has a value of approximately 0.04 (uncorrected for extinction). This value, which would be larger in the absence
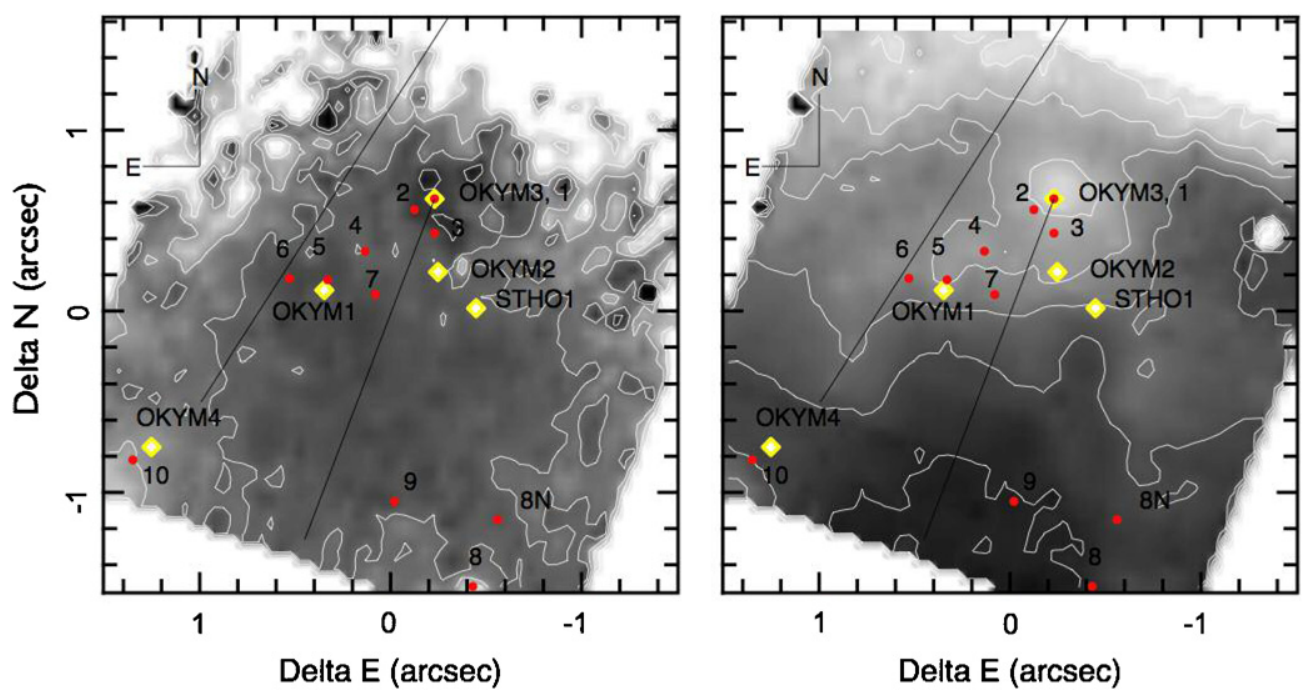

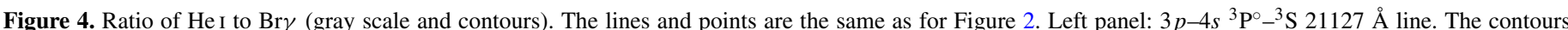

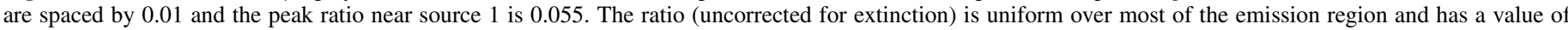

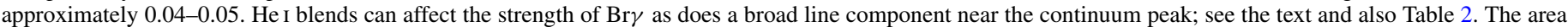

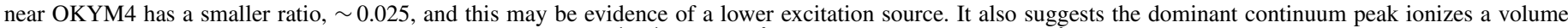

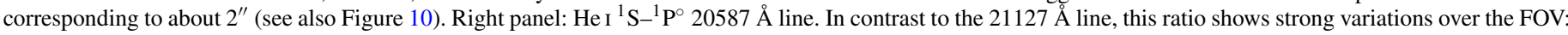
lower values $(\sim 0.25)$ at the continuum peak location and systematically increasing away from the peak to the south $(\sim 0.7)$ in the panel.

(A color version of this figure is available in the online journal.) 

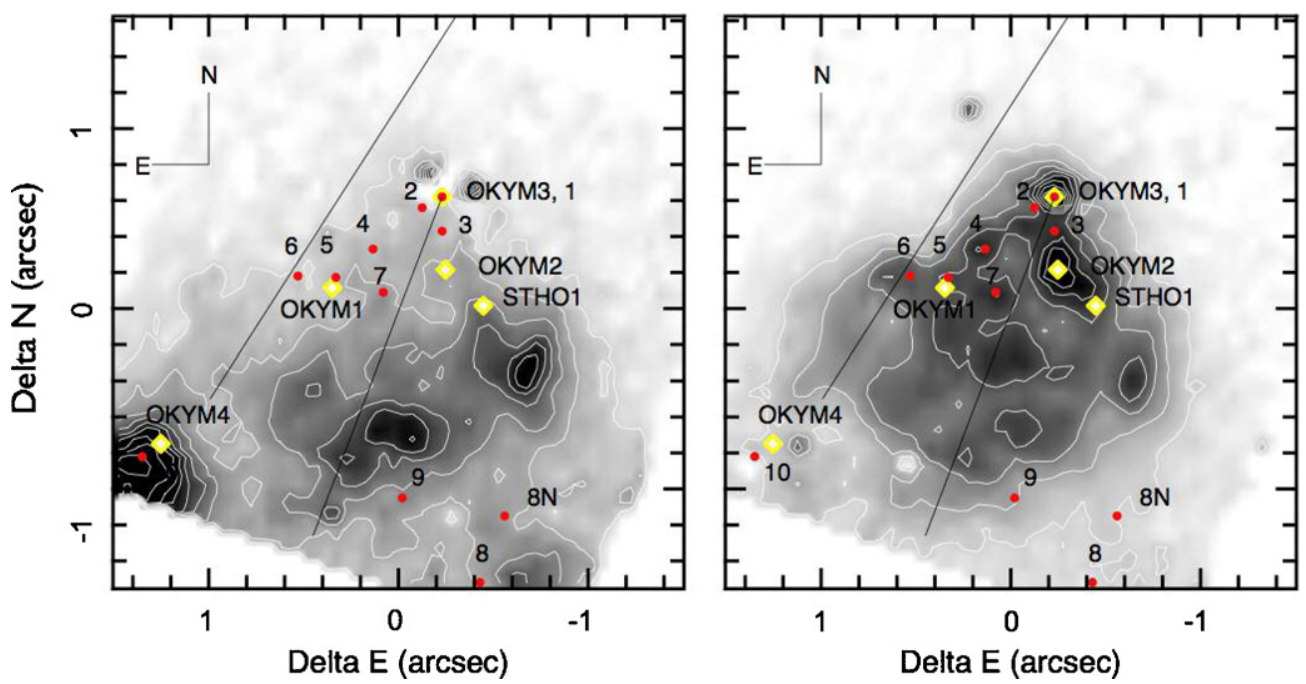

Figure 5. Higher ionization line maps. The lines and points are the same as for Figure 2. [Fe III] $22184 \AA$ is shown on the left, and [Se IV] $22867 \AA$ is shown on the right. The ionization potential of Fe III is lower than that for Se IV showing that the excitation is higher closer to the continuum peak. The peak surface brightnesses are $6.6 \times 10^{-14} \mathrm{erg} \mathrm{cm}^{-2} \mathrm{~s}^{-1} \operatorname{arcsec}^{-2}$ and $1.1 \times 10^{-13} \mathrm{erg} \mathrm{cm}^{-2} \mathrm{~s}^{-1} \operatorname{arcsec}^{-2}$ for [Fe III] and [Se IV], respectively, and the contour intervals are evenly spaced at an interval 10 times less than the peak value.

(A color version of this figure is available in the online journal.)

of extinction, already suggests a hot star (s) with effective temperature $\gtrsim 40,000 \mathrm{~K}$ (Benjamin et al. 1999; Hanson et al. 2002). The stronger He I line,${ }^{1} \mathrm{~S}-{ }^{1} \mathrm{P}^{\circ}$ at $20587 \AA$, is not uniform, however, in comparison to $\mathrm{Br} \gamma$ (see the right panel of Figure 4 and discussion in Section 5.2.3).

Figure 1 shows that strong emission from multiply ionized species exist in the $\mathrm{UCH}$ II region. These include [Fe III], [Kr III], and [Se IV]. The first and last are the strongest lines apart from $\mathrm{He} \mathrm{I}$ and $\mathrm{Br} \gamma$. Figure 5 shows the line maps for two of these lines. The [Fe III] emission is clumpy and peaks on the source OKYM4 while the [Se IV] emission is more intense and somewhat more concentrated to the central "rectangle" or "diamond." The [Se IV] emission has a maximum at the continuum peak $(-0.2,0.6)$ which is unresolved (i.e. compact). The [Fe III] emission also has a local maximum at the continuum peak, but it appears to be affected by one or more bad pixels at the very center. These lines (including [Kr III]) appear to be common in UCH II; see Hanson et al. (2002) and Blum \& McGregor (2008). The ionization potentials of Fe III, Fe IV, $\mathrm{Kr}$ III, Kr IV, Se III, and Se IV are 30.7, 54.8, 37.0, 52.5, 30.8, and $42.9 \mathrm{eV}$, respectively. This suggests that the region with strong [Se IV] emission corresponds with Fe IV (for which there are no identified lines). The range of species seen is consistent with the presence of He I recombination lines (He I ionization potential $=24.4 \mathrm{eV}$ ) and the lack of He II recombination lines $(\mathrm{He}$ II ionization potential $=54.4 \mathrm{eV})$.

Molecular hydrogen emission is also detected (see Figure 1 and Table 1). The strongest line is the $v=1-0 \mathrm{~S}(1)$ line at $21218 \AA$. A map of the line is shown in Figure 6 with the contours of the $\mathrm{Br} \gamma$ emission overlaid. The projection of the $\mathrm{H}_{2}$ emission seems to follow the ionized emission with the peak emission coincident with the $\mathrm{Br} \gamma$ peak. There is a local maximum at the position of the continuum peak as well. The location and excitation of the $\mathrm{H}_{2}$ emission are discussed further in Section 5.4.

\subsection{Gas Kinematics}

Figure 7 shows the measured $\mathrm{Br} \gamma$ line center velocity (in $\mathrm{km} \mathrm{s}^{-1}$ ) for the entire FOV. The mean observed $\mathrm{Br} \gamma$ velocity

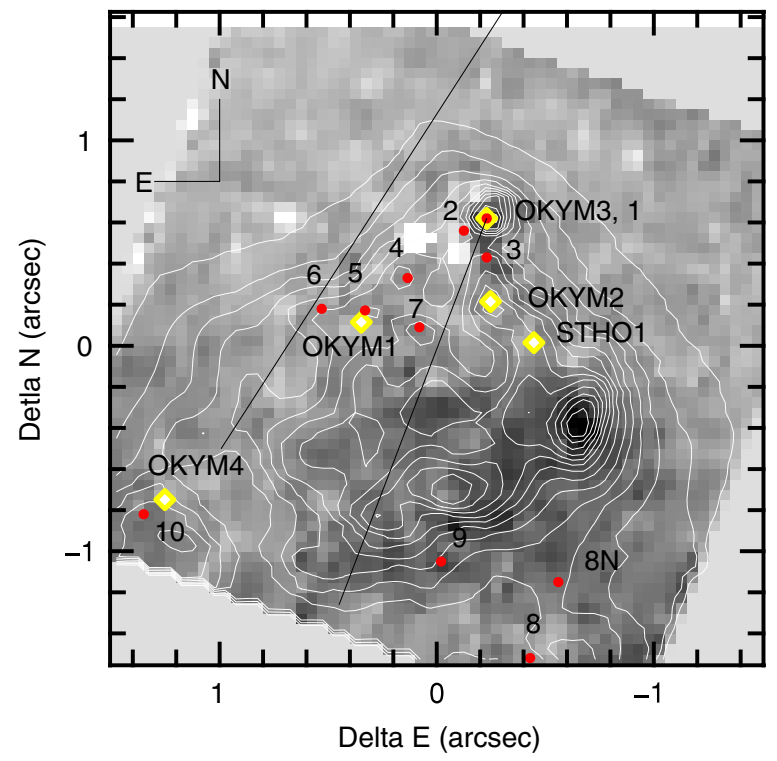

Figure 6. Molecular hydrogen emission map in the v =1-0 S(1) $21218 \AA$ A line. The lines and points are the same as for Figure 2. The emission is clumpy and appears to trace the ionized gas (Figure 3). The peak emission is centered on the peak of the $\mathrm{Br} \gamma$ emission (shown as contours; see Figure 3). An equally strong peak is coincident with the continuum peak (Figure 2). The peak value of $\mathrm{H}_{2}$ emission is $1.7 \times 10^{-14} \mathrm{erg} \mathrm{cm}^{-2} \mathrm{~s}^{-1} \operatorname{arcsec}^{-2}$.

(A color version of this figure is available in the online journal.)

of $-50 \mathrm{~km} \mathrm{~s}^{-1}$ has been subtracted to show relative velocity differences in this map. This mean corresponds to a velocity with respect to the local standard of rest (LSR) $V_{\mathrm{LSR}}=-62.5 \pm$ $5 \mathrm{~km} \mathrm{~s}^{-1}$. The velocities are overplotted with the $\mathrm{Br} \gamma$ emission contours. Each spatial pixel in the $\mathrm{Br} \gamma$ map was fit with a Gaussian profile over a restricted velocity range of -250 to $50 \mathrm{~km} \mathrm{~s}^{-1}$. This choice was made so that the He I line at $21642 \AA$ (see Table 2) did not interfere with the continuum fit. The velocity map shown in Figure 7 was made with a modified version of the Euro3D program (Sánchez 2004) produced by one of us (P.J.M.) and called NF3D.

The $\mathrm{Br} \gamma$ velocity map shows a $\sim \pm 25 \mathrm{~km} \mathrm{~s}^{-1}$ range in velocity centroids. This should be compared with our FWHM 
Table 2

Integrated $\mathrm{He}$ I Lines in $\mathrm{K} 3-50 \mathrm{~A}$

\begin{tabular}{|c|c|c|c|c|}
\hline Line Identification $^{\mathrm{a}}$ & $\lambda_{\circ}(\AA)^{b}$ & $\lambda(\AA)^{\mathrm{c}}$ & FWHM $\left(\mathrm{km} \mathrm{s}^{-1}\right)$ & Ratio to $\mathrm{Br} \gamma^{\mathrm{d}}$ \\
\hline He I $4 s-6 p^{3} \mathrm{~S}-{ }^{3} \mathrm{P}^{\circ}$ & 20430 & 20427 & 79 & $0.0055 \pm 0.0001$ \\
\hline He I $2 s-2 p{ }^{1} \mathrm{~S}-{ }^{1} \mathrm{P}^{\circ}$ & 20587 & 20583 & 67 & $0.6778 \pm 0.0002$ \\
\hline He I $4 p-7 d^{3} \mathrm{P}^{\circ}-{ }^{3} \mathrm{D}$ & 20607 & 20603 & 89 & $0.0067 \pm 0.0002$ \\
\hline He I $3 p-4 s^{3} \mathrm{P}^{\circ}-{ }^{3} \mathrm{~S}$ & 21127 & 21122 & 70 & $0.0462 \pm 0.0001$ \\
\hline He I $3 p-4 s{ }^{1} \mathrm{P}^{\circ}-{ }^{1} \mathrm{~S}$ & 21138 & 21134 & 68 & $0.0110 \pm 0.0001$ \\
\hline He I $4 p-7 s^{3} \mathrm{P}^{\circ}-{ }^{3} \mathrm{~S}$ & 21500 & 21497 & 70 & $0.0012 \pm 0.0001$ \\
\hline He I $4 d-7 f^{3} \mathrm{D}-{ }^{3} \mathrm{~F}^{\circ}$ & 21614 & 21609 & 71 & $0.0250 \pm 0.0002$ \\
\hline He I $4 d-7 f{ }^{1} \mathrm{D}-{ }^{1} \mathrm{~F}^{\circ}$ & 21623 & 21620 & 70 & $0.0100 \pm 0.0002$ \\
\hline He I $4 f-7 g{ }^{1} \mathrm{~F}^{\circ}-{ }^{1} \mathrm{G},{ }^{3} \mathrm{~F}^{\circ}-{ }^{3} \mathrm{G}$ & 21647 & 21642 & 70 & $0.0508 \pm 0.0002$ \\
\hline He I $4 f-7 d{ }^{1} \mathrm{~F}^{\circ}-{ }^{1} \mathrm{D},{ }^{3} \mathrm{~F}^{\circ}-{ }^{3} \mathrm{D}, \mathrm{Br} \gamma ?^{\mathrm{e}}$ & 21655 & 21650 & 70 & $0.0670 \pm 0.0002$ \\
\hline He I $4 d-7 p^{3} \mathrm{D}-{ }^{3} \mathrm{P}^{\circ}$ & 21821 & 21817 & 62 & $0.0044 \pm 0.0001$ \\
\hline He I $4 p-7 d{ }^{1} \mathrm{P}^{\circ}-{ }^{1} \mathrm{D}$ & 21846 & 21842 & 62 & $0.0023 \pm 0.0001$ \\
\hline He I $4 p-7 s{ }^{1} \mathrm{P}^{\circ}-{ }^{1} \mathrm{~S}$ & 22291 & 22287 & 78 & $0.0012 \pm 0.0001$ \\
\hline
\end{tabular}

Notes.

a Atomic emission line identifications were made using the online data base at NIST (Ralchenko et al. 2007) and Peter van Hoof's web page (http://www.pa.uky.edu/ peter/atomic/) that uses energy levels from the NIST database. Most of the lines have wavelengths calculated from the term differences.

b Rest wavelength, vacuum.

c Observed wavelength, mean blue shift is $-3.8 \AA$, or approximately 1.8 pixels.

${ }^{\mathrm{d}}$ Emission lines were extracted from a 2".5 diameter aperture near the center of field of view (see Figure 1). The Br $\gamma$ line map is shown in Figure 3, but the line flux for this ratio was obtained by fitting multiple Gaussians to the He I blends and Br $\gamma$; see the text.

${ }^{\mathrm{e}}$ Emission near the continuum peak is too strong to be due to He I; see the text and Figure 8.

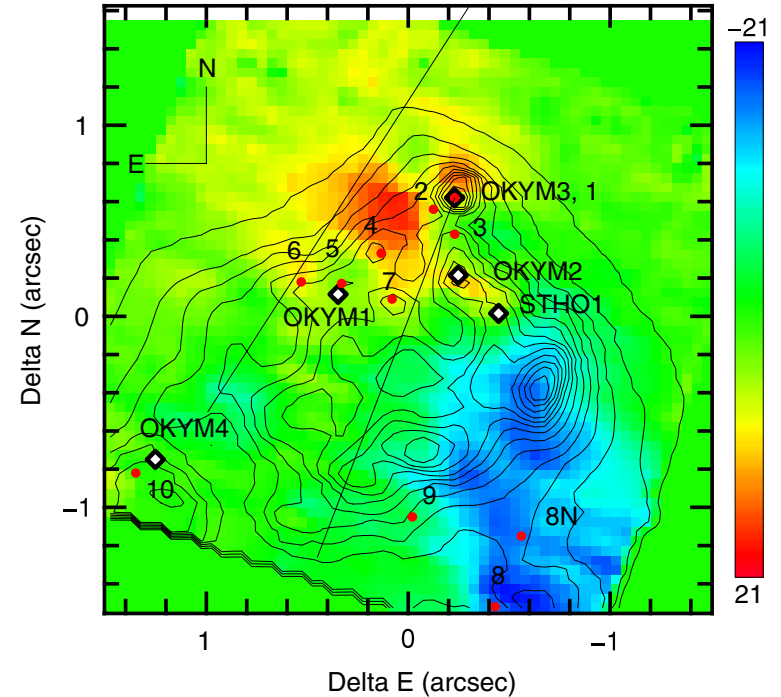

Figure 7. Velocity $\left(\mathrm{km} \mathrm{s}^{-1}\right)$ for the principal component of the $\mathrm{Br} \gamma$ line in each NIFS spaxel derived from the data with a Gaussian fit to the line profile. The labeled lines and points are the same as for Figure 2. The mean $\operatorname{Br} \gamma$ velocity $\left(V_{\mathrm{LSR}}=-62.5 \mathrm{~km} \mathrm{~s}^{-1}\right)$ of the principal component has been removed from the map. The bipolar structure does not line up with any known point source, and the axis does not align with the direction of the large-scale ionized flow detected at radio wavelengths. The peak relative velocities are $-24 \mathrm{~km} \mathrm{~s}^{-1}$ and $+31 \mathrm{~km} \mathrm{~s}^{-1}$, but the color map is "stretched" slightly to show smaller velocities.

velocity resolution of $\sim 60 \mathrm{~km} \mathrm{~s}^{-1}$. Two Br $\gamma$ emission clumps are apparent in Figure 7. These are separated by $1^{\prime \prime}-2^{\prime \prime}$ and oriented approximately at a position angle of $45^{\circ}$. A single Gaussian provides an adequate fit to the $\mathrm{Br} \gamma$ emission over the entire FOV. However, as we show in the following section, there is a broad blue wing to the emission near the continuum peak. In what follows, we call the single Gaussian fit to the bright component of the line "the principal component." When two Gaussians are fit to spaxels near the continuum peak, the effect is to redshift the principal component by a further $\sim 5 \mathrm{~km} \mathrm{~s}^{-1}$ in this vicinity.

The continuum peak is not located near any point of symmetry with respect to the velocity distribution. The nearest possible point source to the apparent center of the velocity distribution (Figure 2) is STHO1 which was not detected in our continuum map. This source is only detected in the mid-infrared [Ne II] map of Okamoto et al. (2003) and not in any continuum image presented here or by Okamoto et al. (2003) or Hofmann et al. (2004).

\subsection{The He I Spectrum and a Broad Bry Component}

The UCH II region K3-50A exhibits a rich He I spectrum in the $K$-band. Table 2 lists the He I lines detected and their properties. Using the online data base compiled by Peter van Hoof ${ }^{5} 13$ lines are identified between 20000 and $22300 \AA$. Other lines redward of $22300 \AA$ may be blended with the H I Pfund series (Figure 1). Multiple He I lines are present in the region around $\mathrm{B} \gamma$. Figure 8 shows spectra extracted from individual spaxels at a typical location $\sim 1^{\prime \prime} .3 \mathrm{SE}$ of the continuum peak (Figure 1, left panel) and a location $\sim 0^{\prime \prime} .2 \mathrm{SE}$ of the continuum peak (Figure 1, right panel). Five Gaussian functions have been fit simultaneously to these features using the spectral analysis program Liner. ${ }^{6}$ Good fits with rms residuals of $<1 \%$ have typically been achieved by assuming that all five lines have the same FWHM as Br $\gamma$ (Figure 1, left panel).

Poorer fits with rms residuals of $\sim 3 \%$ are obtained in the region within $\sim 0$.'5 of the continuum peak where the $\mathrm{Br} \gamma$ line has a strong blue wing (e.g., Figure 1, right panel). The Gaussian component fit to this wing has a central wavelength (which is a fit parameter) that is close to that of the $\mathrm{He}_{\mathrm{I}} 4 f-7 d^{1} \mathrm{~F}^{\circ}-{ }^{1} \mathrm{D}$, ${ }^{3} \mathrm{~F}^{\circ}-{ }^{3} \mathrm{D} 21655 \AA$ line (see Table 2). However, this line is

\footnotetext{
5 http://www.pa.uky.edu/ peter/atomic/; the online database uses data from Ralchenko et al. (2007).

6 Liner is provided by R. Pogge at the Ohio State University. We appreciate Dr. Pogge's support of the Liner package.
} 


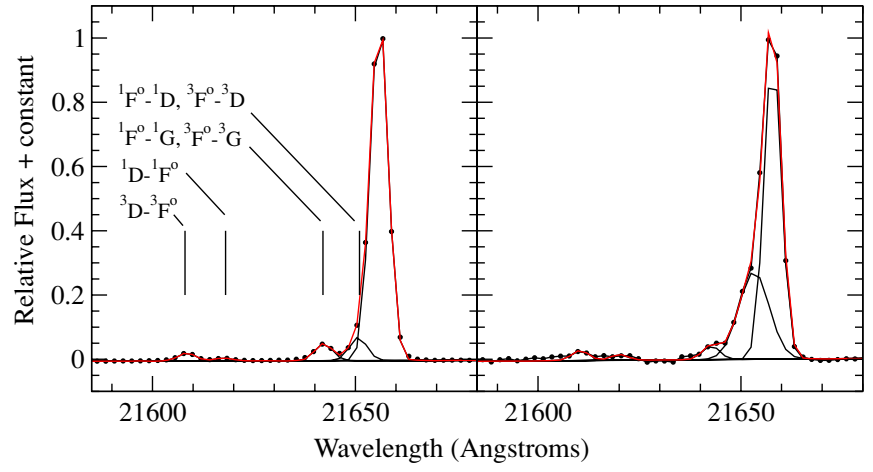

Figure 8. Detail of the line emission in the vicinity of $\mathrm{Br} \gamma$. The vertical hash marks identify He I lines (see Table 2). Five lines were fit simultaneously in each spectrum; see the text. Left panel: emission from a $00^{\prime \prime} 05$ aperture located near Hofmann et al. (2004) source 6. Right panel: emission approximately $0{ }^{\prime \prime} 2$ south and east from the continuum peak in an $\sim 0$ ' 05 aperture. The spectra have been continuum subtracted and scaled to the peak $\mathrm{Br} \gamma$ flux. Each panel shows the observed spectrum in filled circles and the individual lines and continuum as solid black lines. The combined fit is shown as a solid red line. The broad line blended with $\mathrm{Br} \gamma$ in the right panel may be a distinct blue shifted $\mathrm{Br} \gamma$ component. It is much too strong to be due to He I under typical conditions; see the text. The broad line in the right panel is about $120 \mathrm{~km} \mathrm{~s}^{-1}$ FWHM compared to $70 \mathrm{~km} \mathrm{~s}^{-1}$ for the principal component of $\mathrm{Br} \gamma$ (i.e., the bright component).

(A color version of this figure is available in the online journal.)

expected to be too weak to account for the blue wing near the continuum peak. In fact, based on atomic data used in the Cloudy photoionization code (Ferland et al. 1998), the $21655 \AA$ $\mathrm{He}$ I line is expected to be weaker than the $\mathrm{He}$ I $4 f-7 g^{1} \mathrm{~F}^{\circ}-1$ ${ }^{3} \mathrm{~F}^{\circ}-{ }^{3} \mathrm{G} 21647 \AA$ line that is apparent in Figure 1. Better fits are obtained for spaxels near the continuum peak if the FWHM of the blue wing component is allowed to vary. RMS residuals of $<1 \%$ are then obtained with a Gaussian FWHM that is about twice as broad as the principal $\mathrm{Br} \gamma$ line. There is no apparent reason why the $21655 \AA$ A He I line should be anomalously strong and have a width twice that of other He I lines. We therefore conclude that the blue wing on $\mathrm{Br} \gamma$ is due to a mix of a second kinematic component of $\mathrm{Br} \gamma$ that dominates in a small region near the continuum peak and weaker He I emission that occurs over most of the FOV. It is interesting that neither the peak emission nor the width of this second kinematic component is centered on the continuum peak. Instead, this emission forms an arc that is offset SE of the continuum peak by $<0$ '.5.

Whatever the source of the broad component, the contribution of the lines near $21600 \AA$ make a significant contribution to the integrated "Br $\gamma$ " flux. For the spaxel shown in Figure 8, the broad line produces $50 \%$ of the corresponding $\mathrm{Br} \gamma$ flux.

\section{DISCUSSION}

\subsection{Source Positions \& Morphology}

Wynn-Williams et al. (1977) measured the optical, nearinfrared, and mid-infrared positions of $\mathrm{K} 3-50 \mathrm{~A}$ with respect to the radio continuum (Colley \& Scott 1977) using background stars as a reference. The Colley \& Scott (1977) position is consistent with later radio positions including the high angular resolution study of Turner \& Matthews (1984), and thus, to our knowledge, the Wynn-Williams et al. (1977) positions are still the best available. More recent studies have assumed the near and mid-infrared peaks are coincident with the radio continuum peak (Okamoto et al. 2003; Hofmann et al. 2004). WynnWilliams et al. (1977) find that the $2.2 \mu \mathrm{m}$ and $10 \mu \mathrm{m}$ peaks are coincident, within the $\sim 1^{\prime \prime}$ uncertainties, with the radio continuum peak. The optical and $1.6 \mu \mathrm{m}$ peaks are located to the south west of the radio peak, and this is consistent with an extinction gradient across K3-50A as suggested by Frogel \& Persson (1974) and Wynn-Williams et al. (1977) and found also by Howard et al. (1996).

De Pree et al. (1994) observed K3-50A in the radio continuum (15 GHz) and in the $\mathrm{H} 76 \alpha$ recombination line. The angular resolution of these data is about 1 1.5. The continuum appears to be extended along an axis with P.A. (E from $\mathrm{N}$ ) of $160^{\circ}$ and there is a strong velocity gradient from NW to SE along the extended continuum source (see also Roelfsema et al. 1988). This has led to the accepted model of an ionized outflow along the $160^{\circ}$ axis. The NIFS continuum is shown superposed with the De Pree et al. (1994) radio continuum image in Figure 2. The two images have been aligned assuming the radio and $K$-band continuum peaks are coincident. The large difference in scale and angular resolution are apparent. The overlay is instructive for the following discussion, but there are no obvious connections which can be drawn between features in the two maps.

Molecular line studies have been used to argue that there is a rotating torus of material with major axis roughly coincident with and perpendicular to the radio source. Low angular resolution $\mathrm{CO}$ data and modeling of $\mathrm{HCO}^{+}$line emission suggests this material may be rotating about an axis parallel to the outflow axis. De Pree et al. (1994) first drew attention to a possible link between their ionized outflow and the molecular gas traced by CO line emission (Phillips \& Mampaso 1991), though they cautioned that the low angular resolution of the $\mathrm{CO}$ data $\left(30^{\prime \prime}\right)$ and the marginal evidence for a velocity gradient along its major axis made any link between the two structures "premature." Later, Howard et al. (1997) used observations of $\mathrm{HCO}^{+}$to argue for a rotating torus roughly perpendicular to the ionized outflow. The peak lobes of $\mathrm{HCO}^{+}$emission are separated by $8^{\prime \prime}$, so the scale of the rotating molecular torus is larger than the FOV covered by NIFS. The velocity difference measured between the peaks of the $\mathrm{HCO}^{+}$is $\sim \pm 2 \mathrm{~km} \mathrm{~s}^{-1}$, and the best fit to the velocity profile was found to be proportional to the square root of the radius.

Higher angular resolution observations were made by Okamoto et al. (2003) in the mid-infrared (near $10 \mu \mathrm{m}$ ) and by Hofmann et al. (2004) in the $K$ band $(2.2 \mu \mathrm{m})$. The former observations were diffraction limited based on the 0.'4 FWHM obtained on standard stars, but the images of K3-50A do not clearly separate individual objects owing to intense resolved structure/emission and blended point sources. Okamoto et al. (2003) fit various point sources (Figure 2) to their data. The Hofmann et al. (2004) images, on the other hand, were obtained with a speckle imaging technique and are of similar angular resolution as the data presented here. The higher angular resolution and lower background emission result in more identifiable point sources within the central few arcseconds of K3-50A. Okamoto et al. (2003) and Hofmann et al. (2004) conclude that K3-50A contains multiple massive stars; the former through excitation measurements of mid-infrared ionized lines and the latter based on the detection of multiple point sources in the center of the $\mathrm{UCH}$ II region.

The NIFS data confirm various aspects of the earlier work, principally the $K$-band morphology of the point sources and brighter clumps of emission which are dominated by $\mathrm{Br} \gamma$. The 
$K$-band image of Hofmann et al. (2004), the Okamoto et al. (2003) mid-infrared images, and our NIFS images all indicate an abrupt cutoff in emission to the north of the bright continuum peak. The gradient is extremely sharp and the emission drops precipitously in $\lesssim 0^{\prime} .5$ toward the north of the UCH II region. The gradient in extinction was noted in earlier work (e.g., Wynn-Williams et al. 1977) but is seen very clearly in the $J, H$, and $K$ images of Okamoto et al. (2003). The center of the emission shifts systematically to the south. This geometry has been described as an opening cone with the continuum peak at the cone's apex (Hofmann et al. 2004) and the opening toward the south-south east.

The dense molecular torus (Howard et al. 1997) and other overlying material form the cone structure by obscuring ionized material to the north; see Figure 7 of Howard et al. (1997). The constraining effect of the torus may be responsible for helping to shape the ionized outflow (De Pree et al. 1994). The very strong gradient in emission evident in Figure 2 and the $K$-band image of Hofmann et al. (2004) suggest a possible obscuring disk on small scales, but no kinematic or morphological evidence exists to support this picture, and the radio and near-infrared peaks are offset from the center of the torus (see Figure 14 of Okamoto et al. 2003). If the embedded massive star associated with the continuum peak formed from the molecular torus, then it may have separated itself from this "parental cloud."

\subsection{Ionization of the Nebula}

Okamoto et al. (2003) presented ionic line measurements of collisionally excited Ar III, S IV, and Ne II at approximately 9, 11, and $13 \mu \mathrm{m}$, respectively. Ratios of these lines suggest a relatively low excitation, typical of late O-type or early B-type stars. The radio continuum (Turner \& Matthews 1984; De Pree et al. 1994), however, requires more ionizing photons than a single late O-type star produces. Okamoto et al. (2003) thus concluded that multiple stars ionize K3-50A (as discussed above for the NIFS images, we detect continuum sources, Nos. 1, 4-7 at least, which are centered on local peaks of the $\mathrm{Br} \gamma$ emission). Okamoto et al. (2001) made a similar analysis and conclusion for W51 IRS2, also a massive star forming region. But recently, Barbosa et al. (2008) identified the exciting star of W51 IRS2 directly through its near-infrared spectrum, and it is much hotter (approximately O3-O4). For G29.26-0.02, Morisset et al. (2002) find a best-fit nebular model which also has a cooler central source than the observed spectral type (Watson \& Hanson 1997), though their acceptable models do cover a range of $T_{\text {eff }}$ which includes the observed spectral type. The nebular emission in G45.45 + 0.06 was consistent with the observed spectral types found by Blum \& McGregor (2008), but detailed modeling was not done.

A grid of ionization models has been run to analyze the nearinfrared lines presented here in order to further explore the excitation of the $\mathrm{UCH}$ II region. Cloudy ${ }^{7}$ was run on a grid of input parameters which sample a range of ionizing flux and $T_{\text {eff }}$ of the central source as described by Blum \& McGregor (2008). A base line model grid was run with density $10^{4} \mathrm{~cm}^{-3}$ and the Castelli \& Kurucz (2004) atmospheres $(\log (g)=5.0$ and $Z=0$ ). The simulations were run until the temperature into the cloud reached $30 \mathrm{~K}$. A model He atom was used with 10 energy levels to better resolve the level populations for the near-infrared lines. The "_ism" abundance set available in Cloudy was used which has a $\mathrm{He}$ abundance by number relative to $\mathrm{H}$ of 0.10 . In

\footnotetext{
Calculations were performed with version 07.02 of Cloudy, which was last described in detail by Ferland et al. (1998).
}

the following sections, we discuss how the NIFS observations constrain inputs to the models and also what can be inferred from a comparison of the models and observations.

\subsection{1. [Fe III] Lines}

The density used in the Cloudy runs was constrained by the observed ratio of [Fe III] lines. The ratio of [Fe III] $22184 \AA$ to $21457 \AA$ is sensitive to density (Lutz et al. 1993; Bautista \& Pradhan 1998). The observed ratio (see Table 1) is 3.12 and corrected for $\mathrm{A}_{K}$ of 2.17 (Howard et al. 1996) leads to a ratio of 2.8 and a density of approximately $10^{4} \mathrm{~cm}^{-3}$ according to the $7000 \mathrm{~K}$ model of Lutz et al. (1993) or the $10,000 \mathrm{~K}$ model of Bautista \& Pradhan (1998). Bautista \& Pradhan (1998) give densities for the ratio of [Fe III] $22427 \AA$ to $22184 \AA$ as well, but the observed ratio of 0.5 is on the flat part of their curve and gives a low density of $\lesssim 10^{2} \mathrm{~cm}^{-3}$. The small reddening correction would push toward even lower density. All three lines are stronger toward the south in the FOV and weak near the continuum peak; thus the ratios do not constrain the density near the continuum peak. Turner \& Matthews (1984) derived a density of $10^{5} \mathrm{~cm}^{-3}$ in the central few arcseconds from their $2 \mathrm{~cm}$ data; similar radio data were presented by Kurtz et al. (1994) who find a density of $4 \times 10^{4} \mathrm{~cm}^{-3}$. We discuss models for a range of densities below (see Sections 5.2.2 and 5.2.3 and Figure 11).

The [Fe III] line ratios to $\mathrm{Br} \gamma$ reported in Table 1 are similar to those reported by DePoy \& Pogge (1994) in Orion. Though the emission is relatively strong in the K3-50A spectrum, it may be consistent with most of the Fe being condensed onto dust grains as is the case for Orion; similar ratios and physical conditions in Orion are due to a gas phase abundance which is only a percent of the solar iron abundance (DePoy \& Pogge 1994). The distribution of Se IV and Fe III discussed above suggests some of the gas phase iron in K3-50A must be in Fe IV, but there are no Fe IV lines to estimate the abundance in that species (the upper levels of observable lines are generally too high to be excited in H II regions; Bautista \& Pradhan 1998). For the models described below which fit the data best for the excitation of the nebula, most of the Fe is in Fe III and Fe IV. In some cases, the Fe IV fraction is $\sim 90 \%$, but this still requires the bulk of the iron to be condensed on grains if the total abundance is near or above the solar value.

\subsubsection{Recombination Lines and Excitation}

The map of the observed ratio of He I $21127 \AA$ to $\mathrm{Br} \gamma$ is shown in the left panel of Figure 4. The ratio is approximately uniform across the main emission in the center of the image and has a value of between 0.04 and 0.05 . The highest value is 0.055 near the continuum peak, and regions around the periphery have values in the range $0.025-0.03$. The value in the average spectrum of Figure 1 is 0.046 (Table 2) which includes the effect of the blend of lines near $\mathrm{Br} \gamma$. These other lines are not included in the model prediction shown in Figure 9, although their effect is small. The average ratio of 0.046 is consistent with an O-star which has $T_{\text {eff }} \gtrsim 40,000 \mathrm{~K}$ (Hanson et al. 2002). A small correction for extinction gives an intrinsic ratio of 0.051 if we adopt the observed ratio in Table 2 .

Cloudy models were run for a grid of ionizing flux (phi, $\mathrm{s}^{-1}$ $\mathrm{cm}^{2}$ ) versus exciting source $T_{\text {eff }}$. The former parameter was varied from 12.0 to 16.0 dex and the latter varied from $25,000 \mathrm{~K}$ to $45,000 \mathrm{~K}$. The results for our baseline case (density $=10^{4} \mathrm{~cm}^{-3}$ and blister geometry) are given in Figure 9. The ratio along a given contour becomes approximately constant above $T_{\text {eff }}=$ 


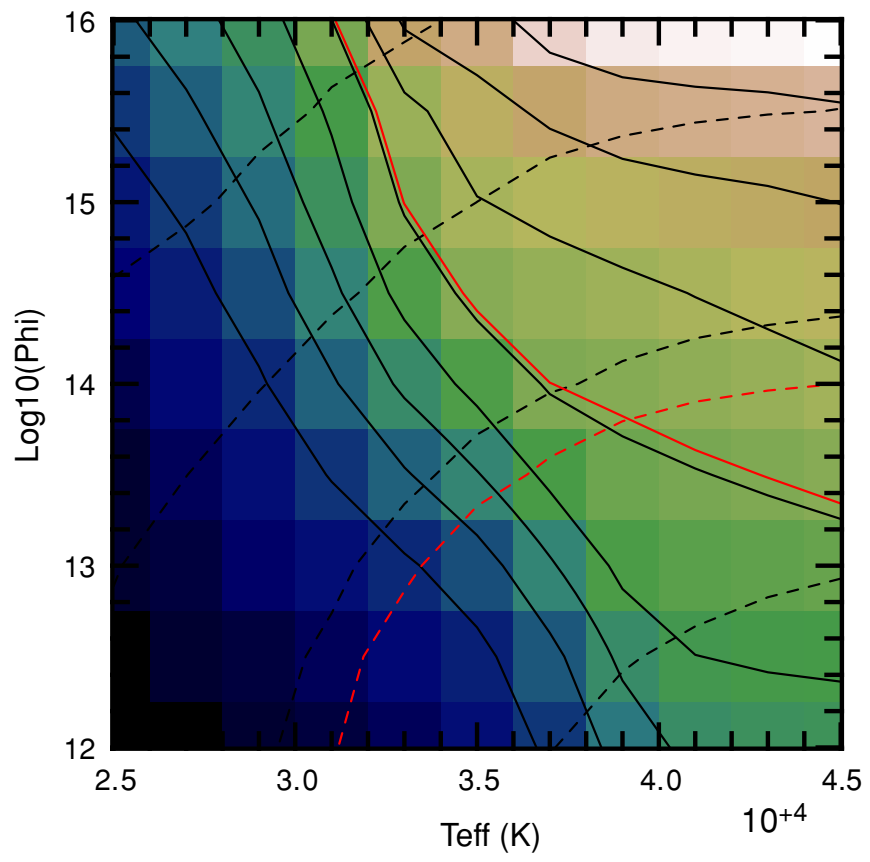

Figure 9. Cloudy ionization model prediction of the He I $21127 \AA$ emission compared to $\mathrm{Br} \gamma$ (color-scale and solid contours) for a model grid of $T_{\text {eff versus }}$ ionizing flux from the central star at the irradiated face of the $\mathrm{H}$ II region. The ratio increases toward higher $T_{\text {eff }}$ and ionizing flux. The contours have values of $0.01-0.08$ and are spaced by 0.01 . The solid red contour is the observed value (Table 2) increased by a few percent to 0.049 to account for differential extinction between the $\mathrm{He} \mathrm{I}$ line and $\mathrm{Br} \gamma$. The dashed contours represent the ratio of $\mathrm{Br} \gamma$ to total dust emission and decrease toward lower $T_{\text {eff }}$ and higher ionizing flux. These contours range from $10^{-7}$ to $10^{-4}$, each separated by a factor of 10 . The dashed red contour is the observed ratio of $1.8 \times 10^{-5}$ (= $3.2 \times 10^{-11} \mathrm{erg} \mathrm{cm}^{-2} \mathrm{~s}^{-1} / 1.8 \times 10^{-6} \mathrm{erg} \mathrm{cm}^{-2} \mathrm{~s}^{-1}$ ) with $\mathrm{Br} \gamma$ corrected for extinction; see the text.

$40,000 \mathrm{~K}$, but the detailed ratio depends on the ionizing flux as well. In Figure 9, the overall emission due to dust compared to the $\mathrm{Br} \gamma$ flux as predicted by Cloudy is also plotted (as dashed contours). The intersection of this contour with the $\mathrm{He}$ I to $\mathrm{Br} \gamma$ contour can give an idea of the true excitation of the nebula under the assumption of a single dominant source (which might not be the case); see Blum \& Damineli (1999) and Blum \& McGregor (2008).

The dust emission was calculated approximately from the spectral energy distribution (SED) shown in Figure 2 of Howard et al. (1997). No attempt was made to correct for the different beam sizes associated with the many distinct data sets which make up the Howard et al. (1997) figure. The most important is the $\sim 100 \mu \mathrm{m}$ point originally reported by Thronson \& Harper (1979) since this is the peak in the SED. The Thronson \& Harper (1979) beam was 50" in diameter and included flux from the nearby $\mathrm{K} 3-50 \mathrm{~B} \mathrm{H}$ II region. On the other hand, higher angular resolution data at $450 \mu \mathrm{m}$ (Thompson et al. 2006) show a very compact source (unresolved at the $8^{\prime \prime}$ beam size) with a similar flux to the SED presented by Thronson \& Harper (1979). Howard et al. (1996) report a Br $\gamma$ flux of $3.2 \times 10^{-11} \mathrm{erg} \mathrm{cm}^{-2}$ $\mathrm{s}^{-1}$ corrected for $A_{K}=2.17 \mathrm{mag}$. We find a integrated dust emission of approximately $1.8 \times 10^{-6} \mathrm{erg} \mathrm{cm}^{-2} \mathrm{~s}^{-1}$ from the Howard et al. (1997) SED. Thus the lower limit to the ratio is $1.78 \times 10^{-5}$.

The intersection of the contours for He I and dust compared to $\mathrm{Br} \gamma$ suggest a $T_{\text {eff }}$ of about $38,000 \mathrm{~K}$ and $\log 10(\mathrm{phi})=$ 13.8. Since the $\mathrm{Br} \gamma$ to dust ratio is a lower limit according the discussion above, the true $T_{\text {eff }}$ would be hotter. The ionization model grid was repeated for a density of $10^{5} \mathrm{~cm}^{-3}$. The results are similar to those in Figure 9 with the intersection of the contours for the observed ratios moving to slightly lower $T_{\text {eff }}$ and higher ionizing flux, i.e., up and to the left in the figure. The formal intersection is at $37,000 \mathrm{~K}$ and $\log 10($ phi $)=14.5$. Changing the geometry from plane parallel (open) to spherical (closed) in Cloudy requires higher incident ionizing flux due to the radiation field dilution by $1 / r^{2}$. In the model grid, the inner radius of the cloud was set to $0^{\prime} .5$ or $5 \times 10^{16} \mathrm{~cm}$. The observed ratios for the spherical case intersect at somewhat higher $T_{\text {eff }}$, $40,000 \mathrm{~K}$, and the corresponding ionizing flux is $\log 10$ (phi) = 14.7-14.4. If the dust emission is too high by a factor of 2 due to beam size mismatches, then the $\mathrm{Br} \gamma$ to dust ratio is too low by a factor of 2 . In this case, the model $T_{\text {eff }}$ could be as high as $45,000 \mathrm{~K}$ for a spherical geometry.

We conclude that the excitation of K3-50A is consistent with a central star whose $T_{\text {eff }}$ is greater than $37,000 \mathrm{~K}$ and less than 45,000 K. This is higher than Okamoto et al. (2003) derive based on line ratios of collisionally excited ions. A similar result is obtained in W51 IRS2 when comparing the results of Okamoto et al. (2001) for line ratios to the recent spectral type obtained for the star located at the position of IRS2 (whose UCH II region is known as W51d); see Barbosa et al. (2008). Evidence is presented below that the geometry may be more open or blister-like (see also Colgan et al. 1991; De Pree et al. 1994), which would lead to $T_{\text {eff }}$ values near $40,000 \mathrm{~K}$.

De Pree et al. (1994) report a Lyman continuum luminosity, $\mathrm{LyC}=2.1 \times 10^{49} \mathrm{~s}^{-1}$ for a distance of $8.7 \mathrm{kpc}$. Adopting $7 \mathrm{kpc}$ (see Section 1), results in a reduced number of $1.3 \times 10^{49} \mathrm{~s}^{-1}$. Kurtz et al. (1994) find LyC $=1.95 \times 10^{49} \mathrm{~s}^{-1}$ for a distance of $8.3 \mathrm{kpc}$, which scaled to $7 \mathrm{kpc}$ gives a similar number. This LyC luminosity is consistent with a single O-type star with effective temperature of approximately $40,000 \mathrm{~K}$ based on the properties for $\mathrm{O}$ stars given by Martins et al. (2005) or 41,000 K if the data from Vacca et al. (1996) are used. Figure 2 shows there are multiple continuum sources (especially in the vicinity of the peak emission), but the excitation of the nebula based on the NIFS spectrum is consistent with a single, dominant star.

\subsubsection{He I $20587 \AA$ Emission}

The strongest He I line in K3-50A is the $20587 \AA$ line (Figure 1). The right panel of Figure 4 shows the line ratio relative to $\mathrm{Br} \gamma$. Comparison to the left panel of Figure 4 indicates the $20587 \AA$ line emission is more variable across the nebula than is the case for the $21127 \AA$ emission. The strong degree of uniformity for the latter suggests the $20587 \AA$ line variations are not due to differential extinction, but instead are due to structure in the nebula. If the variations seen in the right panel of Figure 4 were due only to variations in the line of sight extinction, then this would require a change in $\mathrm{A}_{K}$ of more than $10 \mathrm{mag}$. The continuum map morphology is not consistent with this picture, nor is the average extinction found by Howard et al. (1996) in the central few arcseconds $\left(A_{K} \sim 2\right)$. Finally, the $\operatorname{Br} \gamma$ and He I $21127 \AA$ intensities are near maxima at the position of the continuum peak while the $20587 \AA$ line itself is weak there.

The formation of the He I $20587 \AA$ line is complicated and depends on the physical conditions in the ionized gas (Shields 1993; Ferland 1999). ${ }^{8}$ A large ratio to $\mathrm{Br} \gamma$ is due primarily to high density (Shields 1993) which allows for enhanced collisional population of the $2{ }^{1} \mathrm{P}^{\circ}$ level. However, this level

\footnotetext{
8 The numerical values of the $20587 \AA$ line have changed since the Shields (1993) paper; see Blum \& Damineli (1999) and Ferland (1999).
} 

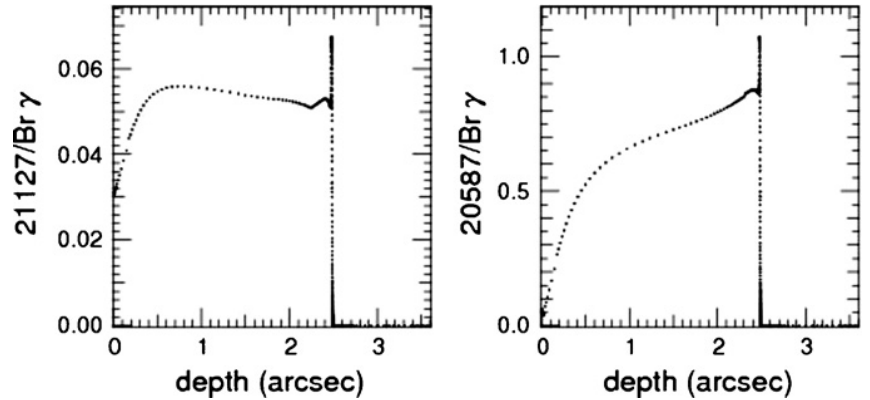

Figure 10. Ratio of $\mathrm{He}$ I to $\mathrm{Br} \gamma$ volume emissivities showing where the lines are formed in the cloud. This plot is for a central object with $T_{\text {eff }}=38,000 \mathrm{~K}$, $\log 10($ phi $)=14$, and density $10^{4} \mathrm{~cm}^{-3}$. Even though the $20587 \AA$ ratio changes significantly within the cloud, average values of the line ratio integrated through the cloud will be relatively large unless the density is low; see the text and Figure 11. The $21127 \AA$ and $20587 \AA$ ratios integrated through the cloud are 0.052 and 0.601 , respectively. The depth coordinate corresponds to the Cloudy model, converting $\mathrm{cm}$ to arcseconds assuming a distance of $7000 \mathrm{pc}$.

population can also depend strongly on $\mathrm{He}$ I L $\alpha 584 \AA$ A resonance fluorescence which is affected by dust absorption of the He L $\alpha$ line and ionization of neutral $\mathrm{H}$ by the $\mathrm{He} \mathrm{L} \alpha$ line since both processes destroy the photons which would otherwise pump the line (Shields 1993). The biggest changes in $20587 \AA$ line formation are expected for a range of central star $T_{\text {eff }}$. The Shields (1993) models were for an average over the nebula; in the present case, the nebula is spatially resolved and clearly is dominated by a point source (or unresolved sources) at one end of the FOV. In Figure 10, the ratio of $\mathrm{He}$ I to $\mathrm{Br} \gamma$ emissivity with depth into the cloud (for a blister model) is plotted for the 21127 and $20587 \AA$ lines and a central source with $T_{\text {eff }}=38,000 \mathrm{~K}$. The $20587 \AA$ line strength changes sharply from inner (close to central source) to outer (far from the central source) positions in the cloud.

Thus, at any given point in the nebula, we would expect a relatively high ratio in the right panel of Figure 4 since the effects shown in Figure 10 would be integrated along the line of sight. The range of observed values for the ratio in the right panel of Figure 4 then suggests that real density variations may exist across the field of view (lower density near the continuum peak). A possible exception to this would be if we were looking along a line of sight such that a slab or blister was illuminated with a geometry whose normal to the slab was in the plane of the sky. But this geometry is inconsistent with the molecular hydrogen morphology (see below, Section 5.4) which suggests a face-on blister geometry.

In Figure 11, the cloudy predictions for the ratios of the $20587 \AA$ and $21127 \AA$ lines to $\mathrm{Br} \gamma$ versus density from Cloudy are plotted. The latter line has an observed ratio of approximately 0.051 (corrected for extinction) and is nearly uniform over the nebula. The former line has an observed ratio (corrected for extinction) that varies from about 0.33 to 0.84 . Figure 11 shows the Cloudy prediction for the case of $T_{\text {eff }}=38,000 \mathrm{~K}$ and $\log 10$ (phi) $=14.0$. These results indicate that the $21127 \AA$ line ratio is nearly constant over a range in density of 100 $100,000 \mathrm{~cm}^{-3}$ while the $20587 \AA$ line changes from 0.38 to nearly 1.0 over the same range. The ratios plotted in Figure 11 are integrated along the line of sight through the nebula in contrast to the ratios plotted in Figure 10.

\subsection{Kinematics}

The kinematics evident in the bright $\mathrm{Br} \gamma$ line (Figure 7) do not fit neatly with any of the previous kinematic data that were

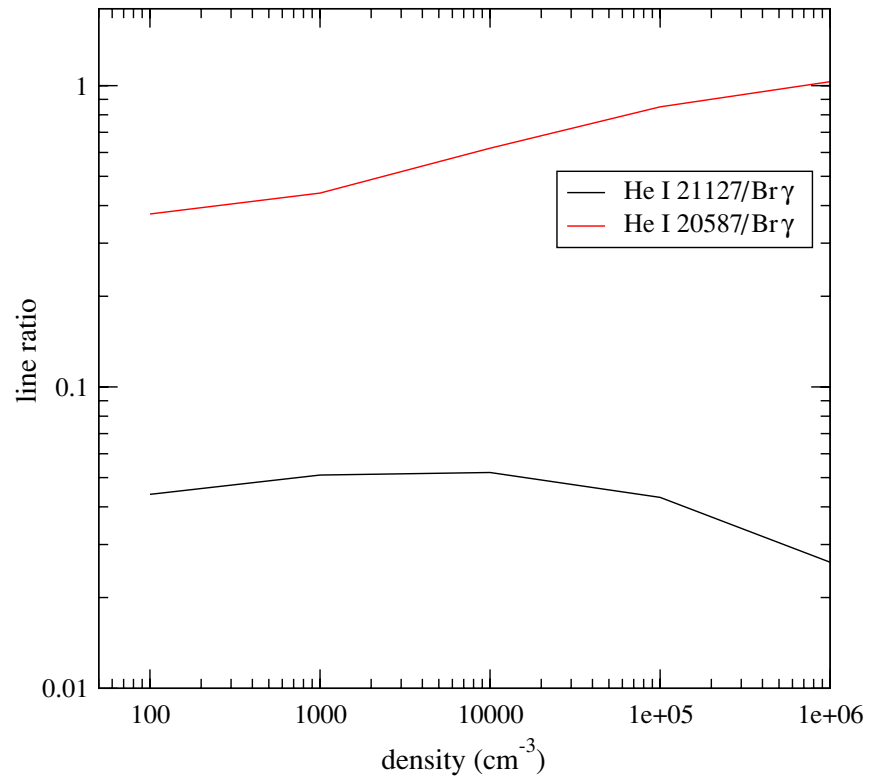

Figure 11. Predicted ratio of $\mathrm{He}_{\mathrm{I}}$ lines to $\mathrm{Br} \gamma$ from Cloudy for a range of nebular densities. These models correspond to a He abundance by number of 0.1 to $\mathrm{H}$. The models were computed with a central star with $T_{\text {eff }}=38,000 \mathrm{~K}$ and the incident radiation field had a flux of ionizing photons, $\log 10(\mathrm{phi})=$ 14.0.

(A color version of this figure is available in the online journal.)

obtained at lower angular resolution. Figure 7 shows a double lobed structure, possibly a flow, with a major axis distinct from any of the larger scale components discussed above. Furthermore, the ordered red and blue shifted material is not well correlated with much of the strong $\mathrm{Br} \gamma$ emission and the point of symmetry of the indicated flow is not coincident with the continuum peak. A possible explanation for the small-scale flow is that it is created by a lower mass protostar in the nascent cluster. This molecular outflow is then ionized by the massive star at the continuum peak. A similar scenario was recently described for the compact high mass star forming region IRS2 in W51 (Lacy et al. 2007; Barbosa et al. 2008).

Another possibility is that the large-scale ionized flow is related to the inner flow. This would require that the outflow is precessing or that details of the interaction of the out-flowing gas on larger scales than the NIFS FOV reorient the flow since the axis of the inner flow does not line up with the axis of the large-scale ionized flow. The contours of the larger flow do, however, appear to twist (De Pree et al. 1994) and Figure 2 clearly shows that more detailed radio (kinematic) observations would be helpful in understanding the transition between the inner scale and larger scale gas kinematics. De Pree et al. (1994) argued that the large-scale ionized flow might result from the expansion of material coming from within a small (few arcsecond) shell. They took as evidence for a shell the high angular resolution $2 \mathrm{~cm}$ map of Turner \& Matthews (1984). The NIFS $\mathrm{Br} \gamma$ morphology appears to match well with the $2 \mathrm{~cm}$ emission, but does not appear shell like (Turner \& Matthews 1984, noted the classification of K3-50A as shell like was problematic). Still, the NIFS data suggest an outflow originating within this small region which is likely confined or directed by the material around it. The large-scale flow has a velocity gradient of about $6 \mathrm{~km} \mathrm{~s}^{-1} \operatorname{arcsec}^{-1}$ while the small-scale flow gradient evident in Figure 7 is $\gtrsim 25 \mathrm{~km} \mathrm{~s}^{-1} \operatorname{arcsec}^{-1}$. The velocity reported for the core of the radio emission is $-33 \mathrm{~km} \mathrm{~s}^{-1}$ with respect to the LSR (De Pree et al. 1994). The integrated $\mathrm{Br} \gamma$ 
emission has $V_{\mathrm{LSR}}=-62.5 \mathrm{~km} \mathrm{~s}^{-1}$ (see Table 1). The redshifted emission in Figure 7 is at $V_{\mathrm{LSR}} \sim-30 \mathrm{~km} \mathrm{~s}^{-1}$. This suggests that we may be only seeing part of radio-emitting gas in the $\mathrm{Br} \gamma$ map and that the red lobe of the $\mathrm{Br} \gamma$ flow is really tracing the bulk of the radio flow; that velocity is presumably the systemic velocity of the radio UCH II region. In this model, all the rest of the $\mathrm{Br} \gamma$ emission is tracing blueshifted gas outflowing from the central source. The spatially unresolved $\mathrm{Br} \gamma$ emission at the continuum peak (see Figure 3 and Section 5.6) also has a $V_{\mathrm{LSR}}$ $\sim-32 \mathrm{~km} \mathrm{~s}^{-1}$ that is close to the systemic velocity defined by the $\mathrm{H} 76 \alpha$ emission.

\subsection{Nebular Structure}

In Section 4.3, a broad component of emission was identified to the blue of the $\mathrm{Br} \gamma$ line (see Figure 8). The position of the line is consistent with one of the He I lines from the $n=7-4$ complex, but the emission is much stronger than expected based on transition probabilities and broader than any other He I line by about a factor of 2. Thus, it is more likely a second $\mathrm{Br} \gamma$ component. The line velocity is about $40 \mathrm{~km} \mathrm{~s}^{-1}$ blue shifted compared to the background or cloud velocity (the green regions in Figure 7). This is larger than the blueshifted velocity structure of the principal component of $\mathrm{Br} \gamma$ shown in that Figure. The width of the broad component is about twice the width of the principal $\mathrm{Br} \gamma$ component $\left(120 \mathrm{~km} \mathrm{~s}^{-1}\right.$ compared to $\left.70 \mathrm{~km} \mathrm{~s}^{-1}\right)$. The broad component is distributed in an arc to the SSE of the continuum source $\left(\sim 0^{\prime \prime} .5\right.$ in length and offset from the peak by $\sim 0^{\prime \prime} .2-0.3$ ) and is confined to this vicinity of the continuum source.

It is not clear how the high velocity, broad component is produced. It may be related to hot gas escaping a tightly constrained volume near the central source. The scale associated with the broad component is small, only $0.007-0.010 \mathrm{pc}$ (for a distance of $7 \mathrm{kpc}$ ). In champagne flows that are produced as the ionizing radiation from the hot star penetrates into a uniform medium (e.g., Yorke et al. 1984), the hot star quickly carves out a larger diameter cavity ( $\gtrsim 0.1 \mathrm{pc})$. Perhaps shocked gas initially flowing parallel to the plane of the sky and then being redirected along our line of sight could explain the broad component. This putative flow might be related to the central source stellar wind.

In any case, gas must escape to the north and eventually form the larger ionized flow seen in the De Pree et al. (1994) radio images. The immediate vicinity of the continuum peak may be filled with low density gas, and this gas may surround (or overlay) higher density material within the projected "cone" which extends to the south of the continuum source. In order to explain the low ratio of $\mathrm{He}$ I $20587 \AA$ to $\mathrm{Br} \gamma$ near the continuum peak, the particular line of sight must contain very little dense ionized gas indicating this area has been cleared away as the hot star emerges from its parent cloud (which remains mostly to the north). The high angular resolution $2 \mathrm{~cm}$ image of Turner \& Matthews (1984) and the molecular maps of Howard et al. (1997) are consistent with clearing of high density material in the vicinity of the continuum peak; both show voids or gaps in the emission. None of the current absolute positions is good enough to place the different wavelength data to better than about 0 .' 5 which is the scale of the structure in our data and the $2 \mathrm{~cm}$ map.

The nebula is seen in projection against $\mathrm{H}_{2}$ emission, primarily in the lower left quadrant where the $\mathrm{Br} \gamma$ emission is strongest (Figure 6) but also (unresolved) at the position of the continuum peak. Three prominent lines of molecular hydrogen are listed in Table 1. The average ratios of the two longer wavelength lines to the $v=1-0 \mathrm{~S}(1) 21218 \AA$ line $(0.42 \pm 0.1,0.23 \pm 0.1$ for $\mathrm{H}_{2} v=1-0 \mathrm{~S}(0)$ and $\mathrm{H}_{2} v=2-1 \mathrm{~S}(1)$, respectively) can be compared to the diagnostic diagram given by Hanson et al. (2002) which shows these same ratios for a large sample of UCH II regions along with several model predictions for shock excited and fluorescent emission. The bulk of the Hanson et al. (2002) sample is consistent with dense $\left(10^{6} \mathrm{~cm}^{-3}\right)$ photodissociation regions (PDR). The results for $\mathrm{K} 3-50 \mathrm{~A}$ place it in the same region of the diagram showing it too is consistent with a dense PDR on the boundary of its ionized nebula. Since we see both the ionized gas and molecular emission, it is likely the $\mathrm{H}_{2}$ emission is behind the ionized gas (a large column of molecular gas in front would obscure our view). If this is the case, then the $\mathrm{UCH}$ II region may be best described as blister like; the hot star has emerged from the parent cloud and is ionizing the face of the molecular material to the south. In this case, the "cone" is really a flattened structure seen face on and lying on the surface of a dense cloud of molecular gas.

\subsection{The Ne, Ar, and S Lines}

As mentioned above, Okamoto et al. (2003) analyzed [Ar III]/ $[\mathrm{Ne}$ II] and [S IV]/[Ne II] ratios in K3-50A. The observed ratios are consistent with other UCH II regions, but inconsistent with simple ionization models. The models predict stronger [S IV]/ [Ne II] than [Ar III]/[Ne II] for a given $T_{\text {eff }}$, but the observations show the average ratios are of similar value and the peak ratios have substantially larger [Ar III]/[Ne II] than [S IV]/[Ne II]. Our grid of models agrees with Okamoto et al. (2003) in the sense that for a given $T_{\text {eff }}$, the [S IV] line ratio is predicted to be stronger.

Martín-Hernández et al. (2002) computed Ar, S, and $\mathrm{Ne}$ abundances for K3-50A from ISO mid-infrared spectra and find abundances which differ from the solar values (and our Cloudy models). In particular, the $\mathrm{Ne}$ abundance compared to $\mathrm{H}$ is approximately solar, while the Ar abundance is a factor of 2 lower than the solar value, and the $\mathrm{S}$ abundance is a factor of 10 lower. However, this large reduction in $\mathrm{S}$ abundance may be (in part) due to the high density in K3-50A depopulating the upper levels producing the $\mathrm{S}$ emission as Martín-Hernández et al. (2002) point out. Detailed models used to derive the $S$ abundance in K3-50A by Afflerbach et al. (1997) give a value only a factor of 2 lower than solar. The ISO spectra were obtained through large apertures $\left(\sim 20^{\prime \prime}\right)$, and the line ratios are smaller than the peak ratios in the higher angular resolution Okamoto et al. (2003) data. The ISO line ratios are nearly equal and have a value of approximately 0.25 . This is comparable to the low end of the range of values obtained by Okamoto et al. (2003). The observed high peak line ratios of Okamoto et al. (2003) ([S IV]/ $[\mathrm{Ne} \mathrm{II}]=3,[\mathrm{Ar} \mathrm{III}] /[\mathrm{Ne} \mathrm{II}]=4$ ) would appear to require lower $\mathrm{S}$ abundance and higher $T_{\text {eff }}$ to be consistent with the Cloudy ionization models, but the precise line ratios do not converge in our grid of models even when adopting the detailed abundances of Martín-Hernández et al. (2002). In particular, reproducing the high line ratio of [Ar III]/[Ne II] requires $T_{\text {eff }}$ to be $45,000 \mathrm{~K}$, the limit of the range based on the analysis of the He I $21127 \AA$ line and the dust emission, and such a model would produce a much too high $[\mathrm{S} \mathrm{IV}] /[\mathrm{Ne}$ II] ratio.

Earlier work based on Kuiper Airborne Observatory observations (Colgan et al. 1991) also did not succeed in fitting the ratios of these infrared lines. The Colgan et al. (1991) data were obtained through apertures approximately $5^{\prime \prime}$ in diameter and 
the line ratios are larger than the ISO values (about unity for both) and somewhat smaller than the Okamoto et al. (2003) peak values. Colgan et al. (1991) and Okamoto et al. (2003) have argued that multiple ionizing sources might explain the observations.

The NIFS data generally (continuum, He I lines, resolved nebular structure) seem to indicate the central few arcseconds are dominated by the continuum peak. Figure 5 shows that the excitation in the nebula is higher toward the continuum peak. The [Fe III] emission is weak in the vicinity of the continuum peak compared to the [Se IV] line. The [ $\mathrm{Kr} I I I]$ line is weak as well and this is consistent with its lower ionization potential than the other species present. In the case of OKYM4 (Figure 2), there may be some evidence for lower excitation; Okamoto et al. (2003) preferred a late O or B star for this source. The left panel of Figure 4 shows a dip in the He I $21127 \AA$ to $\mathrm{Br} \gamma$ ratio at the position of OKYM4 (the ratio is about 0.025 ). This source is about $2^{\prime \prime}$ away from the continuum peak and this is roughly the scale of an associated Stromgren sphere for the central star (Figure 10). Further, the [Fe III] emission peaks at the location of this source. Indeed, the emission morphology of the line maps suggest the boundary of the H II region which is principally due to the continuum peak is on this same scale. If this is correct the "cone" morphology arises from dust obscuration to the north and the ionization boundary of the nebula to the south. The main nebula is largely excited by the continuum peak, and OKYM4 may be locally internally excited. The compactness of OKYM4 then suggests the source is very young and/or highly constrained.

Lower mass OB stars should not greatly affect the structure within the "cone," however. Colgan et al. (1991) and Okamoto et al. (2003) argued that the low average [Ar III]/[Ne II] and [S IV]/[Ne II] ratios might be due to multiple lower $T_{\text {eff }}$ sources (as may be the case for OKYM4), but a successful model must also explain the detailed ratios including their very high peak ratios near the continuum peak. While we have not been able to produce such a model, higher ratios are more consistent with hotter stars.

\subsection{The Continuum Peak}

In the preceding sections, the structure of the nebula was described, and it was concluded that the central source is a hot star emerging from its natal cocoon. The star is ionizing the cloud to the south in our FOV and produces an intense UCH II region and associated PDR. It was argued that the density in the nebula may be lower in the vicinity of the continuum peak.

The continuum peak (by definition) dominates the $K$-band emission. In addition, $\mathrm{Br} \gamma, \mathrm{He}$ I $21127 \AA$, [Se IV] (the highest ionization line observed), and $\mathrm{H}_{2}$ show unresolved emission peaks (or near peaks) at this location. There is clearly a very compact source of emission here which includes stellar continuum (from multiple sources) re-radiated by hot dust, and possibly line emission from the circumstellar environment. An ionized circumstellar disc is a possible source of the compact emission. Though no kinematic evidence is available to support this possibility, Bik \& Thi (2004) and Blum et al. (2004) presented kinematic evidence of discs around (somewhat less) massive young stars based on the emission profiles of the $\mathrm{CO}$ $v=2-0$ rotational-vibrational bandhead at $22935 \AA$. This line is not detected in $\mathrm{K} 3-50 \mathrm{~A}$. Whatever its geometry, there must be compact circumstellar material (partially) surrounding the central source which is not directly linked (at least now) to the larger resolved UCH II region covered by the NIFS FOV.

\section{SUMMARY}

High angular resolution $(\sim 0$ '.2) $K$-band spectral imaging has been presented for the Galactic UCH II region K3-50A. The data were obtained with $\mathrm{AO}$ and the image slicing integralfield spectrograph, NIFS, at Gemini North. The combination of excellent wavelength coverage, moderate spectral resolution (5160), and high angular resolution provide the most detailed look to date at this massive young stellar object and its immediate environment. The emission morphology matches well with earlier speckle observations.

Spatial and ionization structure are resolved in the $3^{\prime \prime}$ nebula. The He I emission morphology suggests changes in density across the field which are attributed to the break out and clearing phase of massive star birth. The ratio of $\mathrm{He}_{\mathrm{I}} 20587 \AA$ to $\mathrm{Br} \gamma$ varies from about 0.3 to 0.8 and requires that the density near the central source be more than a factor 10 less than farther away. The ionization in the nebula can be traced across the nebula by the varying intensity of [Fe III] and [Se IV] lines.

A grid of ionization models has been run with the code Cloudy (Ferland et al. 1998), and the results of these calculations were compared to the observed He I $21127 \AA$ Aline to $\mathrm{Br} \gamma$ line ratio and dust emission properties. The combination of observed values constrain the nebular excitation to a source with $T_{\text {eff }} \gtrsim 37,000 \mathrm{~K}$. This is somewhat hotter than expected from collisionally excited emission-line ratios in the mid-infrared (Okamoto et al. 2003), but those line ratios, particularly their peak values on scales of $\sim 1^{\prime \prime}$, are not explained by our models (or those of Okamoto et al. 2003).

Despite the high angular resolution of these data which enhances the contrast between background and point source emission, no photospheric lines are detected in the spectrum of the bright continuum source; it remains buried within intense continuum emission. The central source is likely also blended with other point sources as pointed out by other investigators. There is spatially unresolved, bright emission in the lines of $\mathrm{Br} \gamma$, $\mathrm{He}$, and [Se IV] (and possibly [Fe III]) at the continuum peak position and these are attributed to the circumstellar environment of the massive young stellar object.

The NIFS data cube reveals a striking kinematic signature in K3-50A. However, this "flow" is not aligned with the largescale ionized out flow reported in earlier radio continuum and recombination line studies (10's of arcseconds in extent). The NIFS kinematic signature is not symmetric about any detected point source in the NIFS image either. It is possible the bipolar kinematics arise from a lower mass protostar associated with the central star of K3-50A, and the associated material is ionized by the hot central source. A second broad component of $\mathrm{Br} \gamma$ emission is seen within a few tenths of an arcsecond of the continuum peak. This may be hot gas escaping from the cavity resulting from the action of the ionizing radiation on the natal molecular cloud or large-scale torus.

The authors thank Gary Ferland, Ryan Porter, and Peter van Hoof for useful input regarding Cloudy and the He I model. The authors also thank Manuel Bautista for useful conversations regarding the Fe III line ratios and Chris DePree for kindly providing the $14.7 \mathrm{GHz}$ FITS image used in Figure 2. The plots and analysis in this article made use of the Yorick programing language. ${ }^{9}$ This article made use of the SIMBAD database at the CDS. Based on observations obtained at the Gemini Observatory (proposal ID GN-2006A-C-11), which is operated by

\footnotetext{
9 http://yorick.sourceforge.net/.
} 
the Association of Universities for Research in Astronomy, Inc., under a cooperative agreement with the NSF on behalf of the Gemini partnership: the National Science Foundation (United States), the Science and Technology Facilities Council (United Kingdom), the National Research Council (Canada), CONICYT (Chile), the Australian Research Council (Australia), CNPq (Brazil), and SECYT (Argentina).

Facilities: Gemini:Gillett ()

\section{REFERENCES}

Afflerbach, A., Churchwell, E., \& Werner, M. W. 1997, ApJ, 478, 190

Barbosa, C. L., Blum, R. D., Conti, P. S., Damineli, A., \& Figuerêdo, E. 2008, ApJ, 678, L55

Bautista, M. A., \& Pradhan, A. K. 1998, ApJ, 492, 650

Benjamin, R. A., Skillman, E. D., \& Smits, D. P. 1999, ApJ, 514, 307

Bik, A., \& Thi, W. F. 2004, A\&A, 427, L13

Black, J. H., \& van Dishoeck, E. F. 1987, ApJ, 322, 412

Blum, R. D., Barbosa, C. L., Damineli, A., Conti, P. S., \& Ridgway, S. 2004, ApJ, 617,1167

Blum, R. D., \& Damineli, A. 1999, ApJ, 512, 237

Blum, R. D., \& McGregor, P. J. 2008, AJ, 135, 1708

Castelli, F., \& Kurucz, R. L. 2004, arXiv:astro-ph/0405087

Colgan, S. W. J., Simpson, J. P., Rubin, R. H., Erickson, E. F., Haas, M. R., \& Wolf, J. 1991, ApJ, 366, 172

Colley, D., \& Scott, P. F. 1977, MNRAS, 181, 703

DePoy, D. L., \& Pogge, R. W. 1994, ApJ, 433, 725

De Pree, C. G., Goss, W. M., Palmer, P., \& Rubin, R. H. 1994, ApJ, 428, 670

Ferland, G. J., Korista, K. T., Verner, D. A., Ferguson, J. W., Kingdon, J. B., \& Verner, E. M. 1998, PASP, 110, 761

Ferland, G. J. 1999, ApJ, 512, 247

Frogel, J. A., \& Persson, S. E. 1974, ApJ, 192, 351

Hanson, M. M., Luhman, K. L., \& Rieke, G. H. 2002, ApJS, 138, 35

Harris, S. 1975, MNRAS, 170, 139

Hofmann, K.-H., Balega, Y. Y., Preibisch, T., \& Weigelt, G. 2004, A\&A, 417, 981
Howard, E. M., Koerner, D. W., \& Pipher, J. L. 1997, ApJ, 477, 738

Howard, E. M., Pipher, J. L., Forrest, W. J., \& de Pree, C. G. 1996, ApJ, 460, 744

Kohoutek, L. 1965, Bull. Astron. Inst. Czech., 16, 221

Kurtz, S., Churchwell, E., \& Wood, D. O. S. 1994, ApJS, 91, 659

Lacy, J. H., et al. 2007, ApJ, 658, L45

Lutz, D., Krabbe, A., \& Genzel, R. 1993, ApJ, 418, 244

Martín-Hernández, N. L., et al. 2002, A\&A, 381, 606

Martins, F., Schaerer, D., \& Hillier, D. J. 2005, A\&A, 436, 1049

McGregor, P. J., et al. 2003, SPIE, 4841, 1581

Messineo, M., Petr-Gotzens, M. G., Schuller, F., Menten, K. M., Habing, H. J., Kissler-Patig, M., Modigliani, A., \& Reunanen, J. 2007, A\&A, 472, 471

Morisset, C., Schaerer, D., Martín-Hernández, N. L., Peeters, E., Damour, F., Baluteau, J.-P., Cox, P., \& Roelfsema, P. 2002, A\&A, 386, 558

Okamoto, Y. K., Kataza, H., Yamashita, T., Miyata, T., \& Onaka, T. 2001, ApJ, 553,254

Okamoto, Y. K., Kataza, H., Yamashita, T., Miyata, T., Sako, S., Takubo, S., Honda, M., \& Onaka, T. 2003, ApJ, 584, 368

Persson, S. E., \& Frogel, J. A. 1974, ApJ, 188, 523

Phillips, J. P., \& Mampaso, A. 1991, A\&AS, 88, 189

Ralchenko, Yu., Jou, F.-C., Kelleher, D. E., Kramida, A. E., Musgrove, A., Reader, J., Wiese, W. L., \& Olsen, K. 2007, NIST Atomic Spectra Database (version 3.1.2) (Gaithersburg, MD: National Institute of Standards and Technology) (online, available: http://physics.nist.gov/asd3 [2007, August 24])

Roelfsema, P. R., Goss, W. M., \& Geballe, T. R. 1988, A\&A, 207, 132

Sánchez, S. F. 2004, Astron. Nachr., 325, 167

Shields, J. C. 1993, ApJ, 419, 181

Thompson, M. A., Hatchell, J., Walsh, A. J., MacDonald, G. H., \& Millar, T. J. 2006, A\&A, 453, 1003

Thronson, H. A., Jr, \& Harper, D. A. 1979, ApJ, 230, 133

Turner, B. E., \& Matthews, H. E. 1984, ApJ, 277, 164

Vacca, W. D., Garmany, C. D., \& Shull, J. M. 1996, ApJ, 460, 914

Watson, A. M., \& Hanson, M. M. 1997, ApJ, 490, L165

Wynn-Williams, C. G., Matthews, K., Werner, M. W., Becklin, E. E., \& Neugebauer, G. 1977, MNRAS, 179, 255

Yorke, H. W., Tenorio-Tagle, G., \& Bodenheimer, P. 1984, A\&A, 138, 325 